Tadeusz Milewski

\title{
La structure de la phrase \\ dans les langues indigènes de l'Amérique du Nord
}

$\S 1$. Les ressemblances structurales parmi les langues caucasiques, paléoasiatiques et américaines signalêes par N. F. Jakovlev ${ }^{1}$ ) et I. I. Meščaninov ${ }^{2}$ ) méritent d'être étudiées plus en détail. Comme étude préliminaire je voudrais analyser en ce lieu la structure de la proposition simple dans les langues indigènes de l'Amérique du Nord et surtout mettre en relief, parmi les constructions syntaxiques, celles que l'on pourrait comparer avec les constructions analogues des langues asiatiques.

§. 2. Il y a dans toutes les langues du monde des moy ens conventionnels pour exprimer les relations abstraites entre le sujet, l'objet et le prédicat, entre le possesseur et la chose possédée, etc. Ces moyens appartenant au systeme traditionnel de la langue constituent le schème de la proposition. Voici le schème de la proposition transitive du latin Marcus necat leonem: $(S$-nom. $\left.)-(P-s)-(O \text {-acc. })^{3}\right)$. Le sujet est marqué ici par la désinence du nominatif -s, l'objet: par la finale de l'accusatif $-m$ et le prédicat contient le sujet pronominal de la $3^{\mathrm{e}}$ p. sous forme de la terminaison - $t$. Le schème

1) N. F. Jakovlev, Anciens liens des langages du Caucase, de l'Asie et de l'Amérique, Académie des Sciences, URSS, Travaux de l'Institut d'ethnographie N. N. Mikloukho-Maklaï. Nouvelle Série II, Moscon 1947, p. 196-204 (en russe).

$\left.{ }^{2}\right)$ Академик Н. Н. Мещанинов, Глагол, Академия Наук СССР Институт Яззыка и. Мышления им. Н. Я. Марра, Москва-Ленинград, 1949. Стр. 197.

$\left.{ }^{3}\right)$ Abréviations: $S=$ sujet nominal, $A=$ agent nominal, $O=$ objet nominal direct, $\mathrm{O}^{\prime}=$ objet nominal indirect; $s=$ sujet pronominal, $a=$ agent pronominal, $o=$ objet pronominal direct, $o^{\prime}=$ objet pronominal indirect; $P r=$ possesseur, $P e=$ la chose possédée, $p o s=$ pronom possessif; - l’ordre des mots libre, + l'ordre des mots grammatical. 
de la proposition intransitive n'en diffère que par l'absence de l'objet: (S-nom. $)$ - $(P-s)$, p. ex. Marcus dormit. Dans le groupe nominal domus Marci le possesseur est caractérisé par la finale du génitif $-i$ et le nom de la chose possédée est dépourvu des signes morphologiques: $(P e)-(P r-g e ́ n$.$) .$

La structure de la proposition latine est excentrique, car chaque membre y est caractérisé par les moyens, qui définissent son rôle syntaxique, chacun en est constitutif. Cette structure s'oppose à la structure concentrique, où le prédicat unique membre constitutif de la phrase - détermine la fonction des autres membres. En même temps le latin est une langue subjective, car la forme du nom dans la proposition intransitive est identique avec la forme du sujet de la proposition transitive. Ce type linguistique s'oppose aux langues objectives dans lesquelles le nom de la proposition intransitive a la même forme que l'objet de la proposition transitive. Dans les langues subjectives le sujet grammatical apparaît dans les propositions transitives: $(S)-(O)-(P)$ et intransitives: $(S)-(P)$ et l'objet dans les propositions transitives seulement. Dans les langues objectives il n'y a pas de sujet grammatical, mais dans les propositions transitives apparaissent l'agent et l'objet: $(A)-(O)-(P)$ et dans les propositions intransitives l'objet tout seul: $(O)-(P)$. Il faut encore souligner qu'en latin la structure de la proposition est basée sur le système des cas, c.-à-d des formes nominales qui sont employées dans le sy. stème de la langue pour indiquer en même temps les relations abstraites et les directions concrètes, cf: l'accusatif de l'objet video Romam et l'accusatif de la direction eo Romam ${ }^{4}$ ). Dans les langues américaines la proposition est construite tout autrement ${ }^{5}$ ):

4) Cf. L. Hjelm sl e.v, La catégorie des cas I, 1935; II 1937; et J. K u ry ło w i c z, Le problème du classement des cas, Bulletin de la Société Polonaise de linguistique, fas. IX, Cracovie 1949, p. 20-43.

$\left.{ }^{5}\right)$. Cf. Handbook of American Indian Languages by Fran z B o a s Smithsonian Institution, Bureau of American Ethnology, Bulletin 40, Part 1, Washington 1911, abréviation B., et Linguistic Structures of Native America by $\mathrm{H}$ a r r y $\mathrm{H}$ o i jer and others, Viking Fund Publications in Anthropology, 
§ 3. La structure de la proposition dans la plupart des langues de l'Amérique du Nord est concentrique. Les langues excentriques - qui n’apparaissent que sur la côte du Pacifique et dans les pays voisins (Colombie britannique, Californie et Arizona du nord-ouest) - appartiennent pour la plupart à la famille penutia (yokuts, maidu, nass, tsimshian), quoiqu'il y a des langues de la famille penutia qui sont concentriques (chinook) et des langues excentriques qui ne sont pas penutia (haida, kwakiutl, hopi). La structure de la proposition dans les langues excentriques de l'Amérique du Nord ne s'appuie pas sur le système de cas comme la proposition latine. Au contraire elle est construite au moyen du système des formes syntaxiques de mots indépendants (yokuts, maidu, hopi, haida) ou au moyen des constructions cycliques (kwakiutl, nass, tsimshian). Dans les langues du premier type chaque membre de la proposition est caractérisé par ses affixes ou par sa position, pourtant ces moyens sont purement abstraits et n'indiquent aucune direction. Nous allons examiner d'abord les langues de ce type.

$\S 4$. La structure la plus excentrique de la proposition caractérise le yokuts ${ }^{6}$ ), une langue de la famille penutia parlée en Californie centrale. Le prédicat marqué par les suffixes du temps, de l'aspect, de la voix et du mode n'y connaît pas la catégorie de la personne. Il se caractérise soimême, c'est son unique fonction syntaxique et pareillement fonctionnent les autres membres de la proposition, qui tous sont constitutifs au même degré. L'ordre des mots est libre. Le sujet est marqué par la finale zéro et l'objet par les désinences - $a,-i n,-o$ ou zéro. Ces formes ne sont pas des cas, parce qu'ils n'indiquent pas de direction, par conséquent nous n'avons pas en yokuts le nominatif et l'accusatif, mais le subjectif et l'objectif. Le sujet avec la finale du subjectif zéro, le prédicat

Number Six, New York 19:6, abréviation H. Le classement généalogique des languages américains: d'après E. Sapira, Central and North American Languages, Encyclopaedia Britanica V, p. 138-141 (1929).

${ }^{6}$ ) The Yawelmani Dialect of Yokuts by Stanley S. Newman, $H_{\text {., }}$ p. $222-248$. 
impersonnel et l'objet marqué par la terminaison de l'objectif voici la proposition transitive en yokuts: $(S)-(P)-(O$-obj. $)$, p. ex. 'ama' binethin kay' $w$ - $a$ 'he asked Coyote' ('ama' 'he' pronom indépendant de la $3^{\mathrm{e}} \mathrm{p}$. au subjectif avec la désinence zéro; binethin 'asked' prédicat impersonnel, kay'w-a objectif en - a de kay'iw 'Coyote'; H. 245). La proposition intransitive n'en diffère que par l'absence de l'objet. Donc l'emploi de l'objectif est limité à la proposition transitive, tandis que le subjectif fonctionne comme sujet dans les propositions transitives et intransitives, comme prédicat nominal et comme substantif dans un emploi absolu. Ainsi dans l'opposition objectif : subjectif, l'objectif comme moins répandu a - d'après la loi Zipf-Troubetzkoy - la fonction d'un terme caractérisé, positif, et le subjectif la fonction d'un terme non caractérisé, négatif. Grâce à ce fait le subjectif, le térme négatif de l'opposition, est caractérisé par la finale zéro et l'objectif comme terme positif a la désinence positive $-a$, in ou - $o$. Dans le groupe nominal le possesseur est caractérisé par la finale -in du possessif (pos.) et le nom de la chose possédée est dépourvu d'un signe morphologique: $\left(P_{r}\right.$-pos. $)-(P e)$ p. ex. ta kay'w-in t'ulos 'that (is) Coyote's bow' (t'ulos 'bow', H. p. 246). Comme en latin, en yokuts la forme du possesseur diffère de la forme de l'objet direct. L'objet indirect est formé en yokuts au moyen de la désinence - ni, p. ex. 'angi nan k'ac'-ni wa-nen 'will he give me obsidian?' (nan 'me' objet direct; k'ac'-ni 'obsidian' objet indirect; H., p. 247). La même désinence - $n i$ indique diverses fonctions adverbiales du nom, p. ex. 'ama' taw pana.hin kay'w(a.)-ni 'and there he arrived with Coyote' l'ama' he' sujet, ta-w 'there' locatif, kay'w(a.)-ni 'with Coyote', H. 24z].

La structure de la proposition en yokuts purement excentrique diffère de la structure de toutes les autres langues de l'Amérique du Nord. En maidu ${ }^{7}$ ), une autre langue de la famille penutia parlée dans le Nord-Est de la Californie, le prédicat forme le centre de la phrase. ll est caractérisé ici non seulement à l'aide des suffixes du mode et du temps mais aussi par les désinences personnelles indiquant le sujet pronominal.

7) Maidu, by Roland B. Dixon, B., p. 679-734. 
Le sujet nominal est caractérisé en maidu par le suffixe -m qui est la marque de l'apposition. Dans cette fonction $-m$ apparaît comme finale du membre déterminant des composés nominaux (p: x. sü'-m+bukü 'dog-tail', sü 'dog', bu'ku tail', B. 692), comme marque des appositions adjectivales et enfin comme désinence du sujet, qui dans ce système doit être interprété comme apposition de la terminaison pronominale du prédicat. Contrairement au sujet, qui forme avec le prédicat un groupe syntaxique compact, l'objet marqué par la finale zéro est indépendant, car il ne peut être l'apposition du prédicat n'ayant pas d'affixes objectifs. Le sujet caractérisé par la désinence - $m$ comme apposition du prédicat et l'objet indépendant constituent en maidu le schème de la proposition transitive: $(S-m)-(O)-\left(P_{-s}\right)$, p. ex. süm has maildü dō-kan the dog bit the man (sü-m 'dog' sujet en -m; mai'dü 'man' objet; dō- 'to bit', - $n$ finale de la $3^{e}$ p.; $B$. 215). La proposition intransitive n'en diffère que par l'absence de l'objet: $(S-m)-(P-s$, p. ex. söttim neno'mmaidüm bülsstsoia there was an old couple', lit. 'one old people lived, it is said' (sö'ti-m 'one', $m$ subjectif; neno-m- old', -m la marque du membre déterminant du composé, maidü-m 'man', -m la désinence du sujet; büss- to live', -tso i- it is said le suffixe qui indique que le fait n'est connu qu a la suite des comptes-rendus des autres personnes, $-a$ finale de la $3^{e}$ p.; B. 726). Dans le groupe nominal $(P r-k i) \ldots(P e)$ le possesseur marqué par la finale - ki diffère quant à la forme de l'objet, p. ex. sü'-ki bukü ' 'dog's tail' (sü 'dog', bukü 'tail', B. 712).

Si en maidu le sujet fonctionne comme apposition du prédicat, en hopi ${ }^{8}$ ), une langue du groupe uto-aztèque parlée dans le Nord-Ouest de l'Arizona, au contraire, c'est l'objet qui constitue l'apposition du prédicat. En hopi l'objet apparaît le plus souvent en objectif qui a deux fonctions, car il est employé pour marquer l'objet et le possesseur. Dans le groupe nominal le nom de la chose possédée $(P e)$ apparaît dans l'état construit (c.) qui indique le possesseur pronominal en $3^{\mathrm{e}} \mathrm{p}$. Le possesseur

$\left.{ }^{8}\right)$ The Hopi Language, Torava Dialect by Benjamin Lee Whorf, $H$, p. $158-183$. 
nominal marqué par la finale de l'objectif constitue l'apposition de cette forme pronominale: $\left(P_{r-o b j .}\right)-\left(P e_{-c}.\right)$ p. ex. ta.qat pása’àt 'the man's field', lit. the man his field' (ta.qa 'man', - $t$ finale de l'objectif; pása freld', -àat latin 'eius', la marque de l'état construit de la $5^{\mathrm{e}}$ p.; H. 170). On peut interpréter par analogie l'objet en objectif comme apposition du prédicat et cette conclusion est confirmée par la deuxième forme dans laquelle l'objet apparait en hopi, c.-à-d. la forme de l'apposition adjectivale, p. ex. tav nina 'kills a rabbit' (tav la forme appositionelle du nom ta.vo rabbit, H. 171-2). Dans la proposition tav nina kills a rabbit l'objet tav constitue l'apposition adjectivale du prédicat nina 'kills' et dans la proposition pásayàt tewa 'he saw his field' $(H .170)$ l'objet pás sayàt his field' dans l'objectif fonctionne comme apposition nominale du prédicat tewa 'he saw'. Il n'y a pas d'autre différence entre ces deux constructions que celle qui consiste dans le degré d'indépendance de l'objet qui est plus subordonné dans la première phrase et plus indépendant dans la seconde. Le sujet apparaît en hopi au nominatif qui est caractérisé par la finale zéro et le prédicat contenant les suffixes de la voix, de l'aspect et du mode est impersonnel comme en yokuts. Ainsi dans la proposition transitive en hopi le sujet n'est pas lié formellement aux autres membres tandis que l'objet constitue avec le prédicat un groupe syntaxique compact: $(S)-$ $(O-a b j)-.(P)$, p. ex. ta.qa wekónavnày repámnat the man unbuttoned his coat' (ta.qa 'man' le sujet au nom. sing. à la finale zéro ; wekónavnà- 'coat', $y$ désinence de l'objectif; répa- 'séparation, - $m$ - sulfixe du pluriel, -na- suffixe du causatif, $t$ suffixe du mode; $H$. 181). Le rapport de l'objet et du prédicat est souligné par le fait que le prédicat transitif par sa forme indique le nombre de l'objet, p. ex posna 'drops one' : löhökna 'drops several' (H. 175). Au contraire dans la proposition intransitive $(S)$ - $(P)$ le prédicat est lié au sujet par l'accord en nombre, p. ex. ciroht pé.yáwnemyàn e 'birds fly' (ciró 'bird, -ht

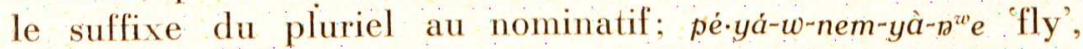
pé.yá(la 'to fly', -nem(a) le suffixe de l'aspect dit "spatial”, -yàle suffixe du nombre indiquant que le sujet intransitif est au pluriel, $-t^{z} e$ suffixe affirmatif; $H$. 176). Par l'accord du 
nombre le sujet et le prédicat d'une proposition intransitive sont liés dans une construction compacte comparable au groupe composé de l'objet et du prédicat dans les propositions transitives. Pour exprimer l'objet indirect on emploie en hopi l'allatif, un cas local qui indique en même temps le but de l'action, le but placé au delà de l'horizon.

$\S 5$. En opposition aux dialectes subjectifs yokuts, maidu et hopi que nous avons analysés, le haida ${ }^{9}$ ) de la famille na-dene parlé dans les îles de la Reine Charlotte (Colombie britannique) et du Prince de Galles (Alaska) est une langue objective. Le prédicat caractérisé en haida par sa position à la fin de la phrase et par des affixes du mode et du temps n'exprime pas la catégorie de la personne, ainsi que le prédicat en yokuts et hopi. Les pronoms indépendants placés hors du prédicat apparaissent dans deux formes: en subjectif (sing $1^{\text {ère }}$ p. $t, 2^{\mathrm{e}}$ p. da, $3^{\mathrm{e}}$ p. la; plur $1^{\text {ère }}$ p. $t ! a l A^{\prime} \dot{n}, 2^{\mathrm{e}}$ p. dal $A^{\prime} \dot{n}$, $3^{\text {e }}$ p. L!) ou en objectif (sing. $1^{\text {ère }}$ p. di, $2^{\text {e }}$ p. dAń, $3^{\mathrm{e}}$ p. la plur. $1^{\text {ère }}$ p. $i L !, 2^{\mathrm{e}}$ p. dal $A^{\dagger} n, 3^{\mathrm{e}}$ p. $L$ !). Dans la proposition transitive l'objet pronominal dans l'objectif (o) est placé à la tête de la phrase; suit l'agent pronominal dans le subjectif $(a)$ et le prédicat transitif (P.tr.) termine la proposition, où l'ordre des mots est fixe: $(o)+(a)+(P . t r)$, p. ex. di dalA'ń Lgaxagî̀lga 'you tire me with your handling' (dī me' objet dans l'objectif; dal $A^{\prime} n$ ' you' agent dans le subjectif; $L$-gaxa-gîl $l$-ga prédicat, $L$ 'by handling' préfixe instrumental, -gaxa- 'weak',- gîl- 'to become', -ga verbe auxiliaire; $B$. 238). Dans la proposition intransitive l'objet pronominal, qui correspond ici au sujet intransitif des langues indo-européennes, apparaît dans l'objectif: $(o)+(P . i n t r)$, p. ex. dì sklîsLdjîtî̀'ga I am truly full' (di objectif de la $1^{\text {ere }}$ p.; sk! issL- to be full', -djît $\hat{t}^{-1}$ - 'truly', -ga to be'; B. 243). Ainsi l'objet pronominal dans les propositions transitives eti ntransitives possède la même forme et occupe la même position, notamment celle en tête de la phrase. Au contraire, l'objet nominal dans la proposition transitive occupe la place après l'agent nominal: $(A)+(O)+(P . t r$.$) , p. ex. Q \bar{a} \mid l$-qons

9) Haida, by John R. Swanton, B., p. 205-283. 


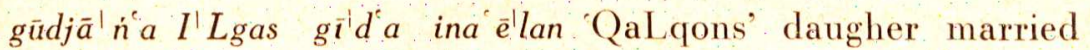
ILgas' son' (gūdjāń 'daughter', - a suffixe possessif; git 'son'; $i^{\prime} n a$ to marry', -êt 'to become'; $B$. 25?). L'objet nominal ne se distingue de l'agent que par sa position et par ses rapports au prédicat. L'agent est syntaxiquement tout-à-fait isolé, mais l'objet constitue avec le prédicat un groupe compact. En haida le prédicat contient souvent un préfixe classificateur qui fait partie d'un système d'environ trente classes qui se distinguent surtout par la forme extérieure des objets. Par conséquent il y a des classificateurs des objets longs, ronds, animés etc. Dans la propositon transitive la classe du prédicat et son nombre (indiqué par quelques radicaux verbaux) s'accorde avec la classe et le nombre de l'objet, p. ex. ga'itgan la sL!gistalyas the pulled out a blood-clot with his finger-nails' (ga'itgań 'bloot-cloot' objet; la 'he' agent pronominal placé après l'objet; $s L !-g \bar{\imath}-s t a-y$-as prédicat transitif, $s L !$ - 'with fingers', -gì-préfixe classificateur pour la classe des matériaux indiquant l'objet, sta- to pull', $-y$ - parfait, -as participe; B. 250). Analogiquement dans la proposition intransitive $(O)+(P$.intr. $)$ la classe et le nombre du prédicat sont régis par l'objet nominal qui remplace le sujet des langues subjectives, p. ex. Inaga'i gaLa îłdAya'gan ga-La ${ }^{\prime} l-d A-y a^{\prime}-g a n-\hat{\imath}$ prédicat, ga- préfixe classificateur pour la classe des objets plats comme la ville, -La'ît- 'five', -dAsuffixe causatif, -ya parfait, -gan passé n'est connu qu'à la suite des comptes-rendus des autres personnes, $-\hat{\imath}$ parfait; B. 231). Le haida ne distingue pas syntaxiquement les adjectifs, du verbe intransitif et les appositions adjectivales, du prédicat. Par conséquent on peut considérer le prédicat (verbal ou adjectival de notre point de vue) comme apposition de l'objet transitif ou intransitif avec lequel il s'accorde en classe et en nombre.

Dans le groupe nominal la relation entre le possesseur et la chose possédée est exprimée par un suffixe possessif (pos.) qui suit le nom du possesseur: $\left(P_{r-p o s}\right)+(P e)$, p. ex.

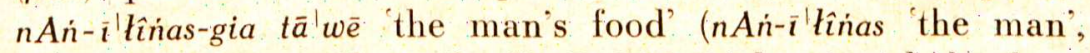
-gia suffixe possessif, tao 'food'; B. 259) ou le nom de la chose 
possédée: $(P r)+(P e-p o s$.$) , p. ex. l'Lgas gīlda ll Lgas' son' (git 'son',$ - a suffixe possessif, $B .257)$.

§6. Dans les langues que nous avons analysées chaque membre de la phrase est caractérisé surtout par sa forme. Au contraire dans les langues cycliques - parlées uniquement en Colombie britannique - la fonction dans laquelle apparaît chaque membre de la proposition est déterminée par le dernier suffixe du membre précédent, ce qui transforme la phrase dans un cycle des formes réciproquement liées. En kwakiutl ${ }^{10}$, la langue cyclique du groupe mos parlée dans la partie sudouest de la Colombie britannique, l'ordre des membres de la propositon est le suivant: prédicat - sujet - instrument (abr. I) ou objet indirect. Ces membres de la phrase peuvent être exprimés par des mots indépendants ou par des suffixes pronominaux, qui apparaissent sous quatre formes, c-à-d. au subjectif $(s)$, à l'objectif (o), à l'instrumental $(i)$ ou au possessif (pos.). Au moyen de ces suffixes on peut exprimer tous les membres de la proposition dans un mot: $(P-s-o-i)$. Le radical prédicatif est suivi ici par les suffixes pronominaux indiquant le sujet, l'objet et l'instrument, p. ex. mix. ̂̀ $n L \bar{o} L a s$ I strike thee with it' (mix- to strike' $=P,-\hat{\imath}^{\top} n L$ - de $-E n L-T=s,-\bar{o} L$ - thee' $=o,-a-$ conjonction, $-s$ with it' $=i ; B .556)$.

La proposition contenant plusieurs mots est construite en kwakiutl a l'aide de quatre séries des sulfixes syntaxiques. Les suffixes de la série subjective (p. ex.-èda, abréviation s') indiquent que le mot suivant possède la fonction du sujet; analogiquement les suffixes objectifs (p. ex. - $x a$, abr. $o^{\prime}$ ) caractérisent le membre suivant de la proposition comme objet et les suffixes instrumentaux (p. ex. -sa, abr. $i^{\prime}$ ) comme instrument ou comme objet indirect. Ainsi la proposition contenant quatre mots est construite en kwakiutl d'après le schème suivant: $\left(P-s^{\prime}\right)+\left(S-o^{\prime}\right)+\left(O-i^{\prime}\right)+(I)$, p. ex. kwexx idèda bEgwän nEmaxa $q ! a \bar{a}$ säsa t!El lwagayo the man struk the sea-otter with the club' (kwêxto strike', $-x$ 'id inchoatif, - $\bar{e} d a=s^{\prime}$ ' bEgwā'nEm 'man', $-x a=o^{\prime}$; $q ! \bar{a} \mid s a$ 'sea otter', $-s a=i^{\dagger} ; \quad t ! E$ 'lwaga to club', -ayō instrument;

${ }^{10)}$ Kwakiutl, by Franz Boas, B, p. 423-557. 
B. 538). Chaque membre de cette proposition n'est caractérisé que par sa position dans la phrase et par le suffixe syntaxique du mot précédent. Le théme du prédicat déterminé par sa position a la tête de la phrase est suivi du suffixe syntaxique subjectif qui caractérise le mot suivant comme sujet. Le sujet, de son côté, est suivi du suffixe objectif, qui détermine le mot suivant comme objet etc.

Dans les propositions contenant deux ou trois mots les membres de la phrase sont exprimés partiellement par les radicaux déterminés par les suffixes syntaxiques des mots précédents et partiellement par les sulfixes pronominaux caractérisés par sa forme: $\left(P-s^{\prime}\right)+(S-o-i)$ ou $(P-s-o)+(O-i)$ etc. (cf. des exemples B. 537).

La proposition intransitive ne differe de la transitive que par l'absence de l'objet et de l'instrument: $\left(P-s^{\prime}\right)-(S)$, p. ex. $g \cdot \bar{a}^{\prime} x$ 'laéda ma'tồ $k w \bar{e}$ 'two persons came, it is said' ( $g \cdot \tilde{a} x$ - 'to come', -la- it is said', -éda ${ }^{\prime} s^{\prime}$; ma lólkwè 'two persons'; B. 537).

Dans le groupe nominal le radical désignant la chose possédée $(P e)$ est élargi par le suffixe syntaxique possessif (pos!.) qui caractérise le mot suivant comme possesseur: $(P e-p o s !)+(P r)$, p. ex. alē'wats!äxs Enư $x^{u}$ g.ț gama éx the hunting canoe of our chief' (alê'wats!äxs- the hunting canoe',-Enu $x^{n}=$ pos!.; g.t'gam- chief"; B. 539).

\$ 7. Au kwakiutl - langue cyclique et en même temps subjective - sopposent nass et tsimshian ${ }^{11}$ ), langues cycliques et objectives parlées tout au long de la côte septentrionale de la Colombie britannique. En nass la structure de la proposition est basée sur l'ordre des mots, l'emploi des sulfixes syntaxiques et l'opposition des trois parties du discours, c.-̀̀-d. les verbes transitifs, les verbes intransitifs (les équivalants des 'verbes intransitifs indo-européens, adjectifs et adverbes) et les noms. Le suffixe syntaxique lie en un groupe deux mots, celui auquel il appartient et celui qui le suit immédiatement. Il y a deux séries de ces suffixes; les suffixes attributifs -Em, - $a$ et les suffixes prédicatifs - $L$ (devant les noms communs)

11) Tsimshian, by Franz Boas, B., p. $283-422$. 
et $-s$ (devant les noms propres). Dans les groupes liés au moyen de suffixes attributif's le membre déterminant devient un attribut dépendant du membre déterminé, au contraire, dans les groupes contenant les suffixes prédicatifs les mots gardent leur indépendance. La structure des groupes de ce dernier type dépend du caractère du premier membre contenant les suffixes $-L$ ou $-s$. Le nom élargi par ces suffixes désigne la chose possédée qui devrait être suivie par le possesseur: $(P e-\mathrm{L})+(P r)$, p. ex. magâlnL K'san 'the mouth of Skeena river' (magâln- 'mouth', $-L$ suffixe syntaxique; $K$ san 'Skeena river'; $B .353$ ). Le verbe intransitif contenant un suffixe syntaxique, prédicatif, fonctionne comme prédicat suivi du sujet: (P.intr.-L) $+(S)$, p. ex. ts'ênL ${ }_{t s}{ }^{\prime} E m e \bar{l} l i x$. 'the beaver entered' ( $t s^{\prime}$ èn 'to enter', $-L$ sulfixe syntaxique, $t s^{3} E m e^{-1} l i x$. 'beaver'; $\left.B .352\right)$. Le verbe transitif élargi par le suffixe $-L$ ou $-s$ et précédé par le sujet est le prédicat qui devrait être suivi de l'objet: $(S)+(P . t r .-L)+(O)$, p. ex. $\bar{a}^{\prime} m L \bar{e}$ wồo na'ksîn 'good you invite your wife!' (am' good'; wôo 'to invite', $-L$ suffixe prédicatif; na'k. 'wife'; $B$. 355). Au contraire, si leverbe transitif contenant le suffixe $-L$ ou $-s$ est placé à la tête de la phrase, il fonctionne comme prédicat et le nom qui le suit est son sujet. L'objet apparaît dans cette construction après le sujet: $(P . t r .-\mathrm{L})+(S)+(O)$, p. ex. wồ $\delta L$ ts'Emè'lix. axt the beaver invited the porcupine' (wôo to invite', - $L$ préfixe prédicatif'; $t s^{\prime} E m e ̄ l l i x$. beaver'; axt 'porcupine'; $B .352$ ). Ainsi le nass est en certain degré une langue objective parce que dans la première de ces constructions transitives l'objet est lié au prédicat dans un groupe syntaxique compact comparable au groupe qui est formé du prédicat intransitif et du sujet: $(S)+(P . t r .-\mathrm{L})+(O)$ analogiquement que $(P$.intr. $-\mathrm{L})+(S)$.

En tsimshian - langue purement objective - la structure de la proposition est basée comme en nass sur l'ordre des mots, l'emploi des suffixes syntaxiques et sur l'opposition des trois parties du discours. Néanmoins, ces moyens ont créé en tsimshian le système beaucoup plus compliqué. Les suffixes attributifs $-E m$ et $-a$ fonctionnent ici comme en nass. Le suffixe prédicatif $-t$ (= nass $-L$ ) n'apparaît en tsimshian que dans des propositions négatives du type: $(P$.intr-1 $)+(O)$, p. ex. awa'lg $E$ 
dzakt wan the deer is not dead yet' (awa'tgE not yet'; dzak dead', - $t$ suffixe syntaxique; wan deer'; $B .365)$, ou dans les propositions négatives du type: $(A)+(P . t r .-1)+(O)$, p. ex. $a^{\dagger} \lg E t$ $d z a^{\prime}$ gut wan he did not kill the deer' ( $a^{\prime} t_{g} E$ not', $t$ 'he' agent; $d z a^{\prime} g$ 'to kill', - $t$ suffixe syntaxique; wan 'deer'; $B .363$ ).

Le plus souvent les propositions du tsimshian sont construites à l'aide de suffixes prédicatifs, positifs qui n'ont pas de correspondants en nass. Il y a deux séries principales de ces suffixes, la série intransitive (p. ex - $-g E$ ) et la série transitive (p. ex. -sgE). Le prédicat transitif placé à la tête de la proposition est élargi par le suffixe intransitif qui caractérise le mot suivant comme agent et l'agent de son côté est élargi par le suffixe intransitif qui caractérise le mot suivant comme objet: $(P \cdot t r .-\mathrm{sgE})+(\mathrm{S}-\mathrm{gE})+(O)$, p. ex. $g \bar{u}^{-} \hat{\imath} \mathrm{s} g E$ huksuli' EnsgEtgE ôlga the hunter hit the bear' (gü to hit', $-s g E$ suffixe transitif; huksul' Ensg hunter', - $g E$ suffixe intransitif; ol bear, -ga signal de la fin de la phrase; B. 356).

Dans toutes les autres constructions syntaxiques n'apparaissent que les suffixes intransitifs et le caractère de la construction dépend du prémier membre qui est toujours suivi du nom. Si le premier terme du groupe est un verbe intransitif, ce nom fonctionne comme objet intransitif: $(P$-intr.-gE $)+(O)$, p. ex. da na-bā'gE ôlga then the white bear ran out of woods' ( $d a$ 'then'; na- 'out of woods', bā- to run', $-g E$ suffixe intransitif; ôl bear'; $B .355)$. Si le premier membre du groupe est un verbe transitif, le nom qui le suit est caractérisé comme objet transitif: $(P . t r .-g E)+(O)$, p. ex. $w a \tilde{a}$ itgE $h \bar{a}$ 'sga he has found the dog' (wai 'to found', -t 'he', $-g E$ suffixe intransitif; $h a ̈ l ' s$ 'dog'; B. 356). Si enfin à la place du premier membre apparaît un nom, nous avons un groupe nominal qui possède en tsimshian une structure double. Dans les groupes du type $(P r-g E)+(n E-P e)$ le nom du possesseur est élargi par le suffixe intransitif $-g E$ et le nom de la chose possédée, avec le préfixe possessif $n E$-, le suit, p. ex. gü $g A$ dzố $g$ at $g E s g E$ qal-ts!a'pgE $n E-w \bar{a} l$ lptga 'who lived in the house of the town' ( $g \bar{u}$ 'who'; $d z \hat{o} g$ to camp', $t 3^{\mathrm{e}} \mathrm{p}$; $g E \operatorname{gg} E$ in'; qal-ts!alb 'town', $-g E$ suffixe intransitif; $n E$ - préfixe possessif, wälb house'; B. 360). Dans un 
autre cas le nom de la chose possédée contient le suffixe intransitif et le nom du possesseur le suit: $\left(\mathrm{nE}-P_{e}-\mathrm{gE}\right)+(P, r)$, p. ex. ada $n E$ wul $n \bar{n} n E-w a a^{\prime} l b s d E$ y'u'ta then I saw the house of the man' (ada 'then'; $n E$ I'; $n \bar{t}$ 'to see'; $n E$ - préfixe possessif, wälb house', $-s d E$ suffixe intransitif équivalent syntaxique du suffixe $-g E$ au subjonctif; $y \cdot \mathbf{u}^{\prime} \cdot t$ 'man'; $B$. 360). Par conséquent le tsimshian est une langue purement objective qui oppose l'agent indiqué par le suffixe transitif du prédicat à l'objet transitif et intransitif déterminés par le suffixe intransitif du mot précédent et au groupe nominal constitué aussi par le suffixe intransitif. Dans l'opposition suffixe transitif : suffixe intransitif le suffixe intransitif comme plus répandu fonctionne comme terme négatif et le suffixe transitif peut être caractérisé comme terme positif.

\$ 8. Les langues excentriques que nous avons analysées n'apparaissent que sur la côte du Pacifique et dans les pays voisins. Toutes les autres régions de l'Amérique du Nord étaient occupées avant l'invasion des blancs par les langues concentriques, dans lesquelles le prédicat grâce à l'incorporation des affixes pronominaux indiquant le sujet et l'objet, ou l'agent et l'objet constitue un schème de la proposition et les termes nominaux de la phrase ne sont que des appositions aux affixes subjectifs et objectifs du prédicat. Ainsi la fonction syntaxique est concentrée dans le prédicat à un degré qui varie d'une langue à une autre. Dans les langues concentriques du premier degré (yuma, fox et eskimo), le sujet et l'objet nominaux ont encore une fonction syntaxique. Ils s'opposent réciproquement par leurs désinences qui marquent à quel affixe du prédicat chacun d'eux fait apposition. Dans les langues concentriques du deuxième degré (delaware, chipewyan, tlingit, chitimacha), l'indépendance du sujet et de l'objet nominaux est diminuée parce qu'ils ne se distinguent que par leur position par rapport au prédicat. Dans les langues du troisième degré (aztèque, dakota), il n'y a pas de moyens syntaxiques pour distinguer le sujet et l'objet qui sont tout-à-fait dépourvus de la fonction syntaxique, mais en même temps cette fonction n'est pas remplie par le prédicat n'ayant pas de moyens pour distinguer 
les autres membres de la proposition. Enfin dans les langues du quatrième degré (hupa, taos, tunica et chinook), le sujet et l'objet nominaux sont également dépourvus de la fonction syntaxique mais cette fonction est remplie par le prédicat qui, grâce à la concordance en genre et en nombre de son affixe subjectif au sujet nominal et de son affixe objectif a l'objet, détermine la fonction d'éléments nominaux de la proposition.

D'un autre point de vue, on peut classer les langues concentriques en synthétiques et analytiques. Dans les langues synthétiques (yuma, chitimacha, taos) n’apparaît qu’une série indifférenciée d'affixes pronominaux qui, dans le prédicat transitif, indiquent en même temps le sujet et l'objet. Au contraire, dans toutes les autres langues concentriques qui ont la structure analytique, fonctionnent au moins deux séries indépendantes d'affixes pronominaux, la série subjective et la série objective.

§9. En tonkawa ${ }^{12}$ ), une langue du groupe hoka parlée jadis dans le Sud-Ouest du Texas, mais provenant de la Californie, apparaissent en même temps les traits de la syntaxe des langues concentriques et excentriques. Si le prédicat tout seul constitue la proposition, il contient en tonkawa - comme dans les langues concentriques - les éléments pronominaux indiquant le sujet et l'objet. L'objet est exprimé par un préfixe ou par l'allongement de la dernière voyelle du thème (sing. 1 ère p. ke- $2^{\mathrm{e}}$ p. allongement, $3^{\mathrm{e}} z$ éro, plur. 1 ̀̀re p. kew-) tandis que le sujet est désigné par un suffixe (p. ex. les suffixes du présent du mode déclaratif: sing. 1 ère p. $-s, 2^{\mathrm{e}}$ p. $-k a, 5^{\mathrm{e}}$ p. zéro, plur. $3^{\mathrm{e}}$ p. -yuk). La proposition entière est concentrée dans un mot formé d'après le schème: $(o-P-s)$, p. ex. ke-ykapo? he hits me' (ke- 'me', ykapo? 'to hit', H. 304).

Dans les propositions contenant le sujet et l'objet nominaux le prédicat est impersonnel et ne se caractérise que par les suffixes du mode et de l'aspect. Les autres membres de la proposition sont marqués par les désinences qui forment quatre séries: indéfinie et définie au singulier et au pluriel.

${ }^{12}$ ) Tonkawa by Harry Hoijer, H., p. 289-311. 
Le sujet est caractérisé par la finale du nominatif (indéf. sing. la, plur. -ka, déf. sing. - pa.la, plur. -?a.ka), l'objet par la désinence de l'objectif (indéf. sing. -lak, plur. -kak, déf. sing. -?a.lak, plur. -?akak) et le possesseur par la terminaison du possessif (indéf.-?an, déf.-?allaan). Ainsi la structure de la proposition transitive en tonkawa est la suivante: (S-sub.) -

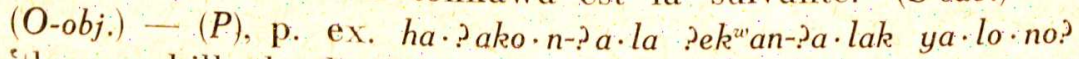

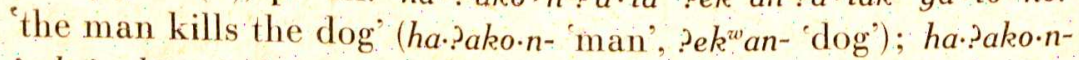
?a.ka? ekw an-?a.kak ya.lo.no? 'the men kill the dogs' (H. 302). La proposition intransitive ne diffère de la transitive que par l'absence de l'objet: (S-sub.) - $(P)$, p. ex. ha. ?ako.n-la ha.no? 'a man goes away" (H. 302).

Le groupe nominal se compose du nom du possesseur contenant la finale du possessif et du nom désignant la chose possédée sans signe morphologique: (Pr-pos.) - (Pe), p. ex. ha.?ako.n-?a.l?an macxanan-?a.la the man's sweetheart' (le premier mot 'man' est dans le possessif en -?a.l?an, le deuxième au nominatif en -3a.la; H. 302).

Ainsi en tonkawa le prédicat qui fonctionne comme une proposition complète possède la structure concentrique, parce qu'il incorpore le sujet et l'objet pronominaux, mais la structure de la proposition à plusieurs mots, caractérisée par le prédicat impersonnel, est excentrique, comme p. ex. en yokuts. La structure du tonkawa est intermédiaire entre ces deux types linguistiques.

$\S 10$. Passons maintenant au yuma ${ }^{13}$ ), langue concentrique du premier degré et en même temps synthétique, qui était parlée dans la partie du sud de la Californie. Le prédicat est caractérisé ici par un suffixe prédicatif, le plus souvent $-k$, et par les préfixes pronominaux. Dans les prédicats transitifs ces préfixes indiquent en même temps le sujet et l'objet, p. ex. de la racine ayú-- 'voir' nous avons ?ańm-ayú--k 'tu me vois', $n$-ayú-k il me voit', ń-ayú-k je te vois', m-ayú-kk il te voit', ?-ayú-k je le vois', m-ayú-k tu le vois', ayú--k il le voit'. Dans les prédicats intransitif's les mêmes préfixes n’indiquent

${ }^{13}$ ) Yuma by A. M. Halpern, H., p. 249-288. 
que le sujet, p. ex de la racine ayér- 'voler' nous avons: ?-ayér-ək 'je vole', m-ayér-ək 'tu voles' ayér-ək 'il vole'. Ainsi les préfixes ?-; m-, zéro, qui dans les prédicats transitifs ont la valeur je le..., tu le..., il le..., dans les prédicats intransitifs n'indiquent que je ..., tu..., il...' (abreviation so).

Le sujet c'est l'apposition du préfixe pronominal élargie par la désinence $-c$; l'objet c'est l'apposition à suffixe zéro. Dans le groupe nominal le nom du possesseur fonctionne comme apposition à suffixe zéro du pronom possessif (p. ex. ?ań- 'mon', mań- 'ton', $\dot{n}$ - 'son'). Ce pronom apparaît comme préfixe du nom de la chose possédée (construction le père sa-maison la maison du père'). Dans la même forme à suffixe zéro on emploie le nom comme apposition d'un autre nom. Ainsi il y a en yuma deux formes de l'apposition nominale, l'apposition en -c et l'apposition à désinence zéro. La première fonctionne comme apposition subjective aux préfixes indiquant 'il me...' il te...' il le...' dans le schème syntaxique: $(\mathrm{S}-\mathrm{c})-(\mathrm{O})-($ so-P.tr.-k), et analogiquement dans les propotions intransitives du type: (S-c) - (so-P.intr-k), p. ex šal'?áy-c ?amé-k the sand is high', lit. 'sand it-is-high' (šal'?áy-c 'sand' sujet au nominatif constitue l'apposition au préfixe zéro du prédicat ?amé-- $k$ 'it-is-high'; $H$. 265). L'apposition à désinence zéro fonctionne dans tous les autres cas, c.-̀̀-d. $1^{0}$ comme apposition objective des préfixes prédicatifs ?-, m-, zéro dans le schème syntaxique: $(O)-($ so-P.tr.-k), p. ex. ń-aván ayú.-k he saw his house', lit. 'his-house he-sees' (H. 286); $2^{0}$ comme le nom du possesseur, c.-à-d. l'apposition du pronom possesif dans le schème syntaxique: $(P r)-($ pos. $-P e)$, p. ex. xatalwé ń-a’ú.v 'coy ote's tobacco', lit. 'coyote his-tobacco' (H. 265); $3^{0}$ comme apposition d'un autre nom, p. ex. xatalwé šal'?áy 'sand coyote', lit. 'coyote sand' ( $H$. 265). Dans l'opposition des formes nominales en -c et en zéro les formes en -c moins répandues fonctionnent comme un terme positif et les formes en zéro sans signe morphologique comme un terme négatif.

\$11. En opposition au yuma, langue synthétique, les autres langues subjectives comme l'aztèque et les dialectes des deux grands groupes linguistiques algonkin et na-dene (athapaskan 
et tlingit), sont analytiques. Elles possèdent deux séries d'affixes pronominaux et ne diffèrent l'une de l'autre que par le degré de la concentration des fonctions syntaxiques dans le prédicat. Le premier degré de cette concentration apparait dans la phrase du fox ${ }^{14}$ ), dialecte du groupe algonkin parlé dans le lowa près de la rive ouest du Mississippi. Il y a en fox deux séries d'affixes pronominaux: la série d'affixes subjectifs indiquant le sujet (sing. 1ère p. ne-, $2^{\mathrm{e}}$ p. ke-, $3^{\mathrm{e}}$ p. animée -wa, inanimée $-w i$, plur tère p. exclusif ne-...pena, inclusif ke-..-pena, $2^{\mathrm{e}}$ p. ke-... $-p w a, 3^{\mathrm{e}}$ p. animée - $w A$ gi, inanimée -ōni) et la série d'affixes subjectifs-objectifs qui expriment en même temps le sujet et l'objet (p. ex. ke-...-ne je te...., ke-..-i tu me..., -äwa il le..., -äwAgi ils les... etc.). Les thèmes transitifs en fonction du prédicat peuvent apparaître dans la forme transitive avec les affixes subjectifs-objectifs ou en intransitif avec les affixes subjectifs. Au contraire les thèmes intransitifs, qui correspondent aux verbes intransitifs, aux adjectifs, aux substantifs et aux numéraux des langues indo-européennes, n’apparaissent que dans la forme intransitive, élargie par les affixes subjectifs. Ainsi il y a en fox trois types de prédicats qui peuvent fonetionner comme propositions complètes: $1^{0}$ Les prédicats intransitifs dérivés des thèmes transitifs ou intransitifs à l'aide d'affixes subjectifs d'après le schéme: $(s-P)$, p. ex ne-pya 'I come', ke-pya you come (sing.)', ou le schéme: $(P-s)$. p. ex. pyä-wa he come' $(B, 817) .2^{0}$ Les prédicats transitifs dérivés des thèmes transitifs à l'aide d'affixes subjectifs-objectifs d'après le schème: $(s-P-o)$, p. ex. ne-wâpAm-āwa I look at him', le schème $(o-P-s)$, p. ex. ke-wâpAme-ne 'I look at thee', ou le schème: $(P$-so), p. ex. wâtAt-Amwa he looks at it' (B. 818-9). $3^{0}$ Les prédicats transitifs à deux objets: direct $(o)$, exprimé par le pronom de la $3^{\mathrm{e}}$ p. - Amaw- $\| A m \bar{o}^{-}$, et indirect $\left(o^{\circ}\right)$ qui est indiqué par les affixes objectifs, p. ex. $\left(o^{\prime}-P-o-s\right)$, cf.

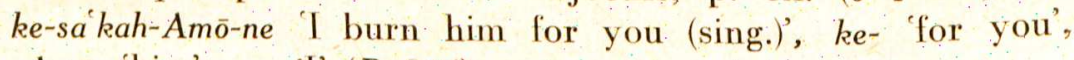
-Amō- him', -ne 'T' (B. 835).

14) Algonquian (Fox), by William Jones (revised by Truman Michelson), B., p. $735-873$. 
Le sujet et l'objet nominaux se caractérisent par leurs désinences et par leur position dans la phrase. Le sujet animé est élargi par la finale du subjectif (sing. - $a$, plur. -gi) et l'objet animé par la désinence de l'objectif (sing. -ni, plur. - i i). Le sujet et l’objet inanimés ont la même finale (sing. - $i$, plur.-ni) et ne se distinguent que par la position par rapport au prédicat. Ainsi la proposition transitive est construite en fox d'après le schème: $(S$-sub. $)+(P$-os $)+(O-o b j$.$) , p. ex. u-tAnemót-$ he-mA'ni wấpAm-ä-niwA ni ma hwäwA'ni his dog looker at the wolf' (u-...-mAni his', tAnemōhe 'dog' sujet; wâpAm- 'to look at', . - ä- signe objectif indiquant $m a^{\prime \prime} h w a ̈ \omega A^{\prime}-n i$ 'wolf', c.-̀̀-d. l'objet du prédicat en objectif caractérisé par la désinence $-n i ; B .832)$. La proposition intransitive ne diffère de la transitive que par l'absence de l'objet: $(S-s u b)-.(P-s)$. Le groupe nominal a la forme d'un nom composé.

\$12. Une structure semblable qu'en fox possède la proposition du delaware ${ }^{16}$ ), une langue du groupe algonkin parlée jadis dans la plus grande partie de la Pensylvanie, NewJersey et Delaware. La différence entre la syntaxe de ces deux dialectes algonkins consiste dans l'identité morphologique en delaware du sujet et de l'objet nominaux qui ne se distinguent que par la position du sujet avant et de l'objet après le prédicat: $(S)+(s-P-o)+(O)$. Grâce à cela il faut considérer le delaware comme une langue concentrique du deuxième degré.

Au même type linguistique appartient le chipewyan ${ }^{17}$ ) parlé entre les lacs Great Slave et Athabasca et sur la Slave river, une langue du groupe septentrional des dialectes athapaskan. Le prédicat y est caractérisé par sa position à la fin de la phrase, par les préfixes du mode, de l'aspect et de la voix et surtout par les préfixes pronominaux subjectifs et objectifs. Le préfixe dans la $5^{\mathrm{e}}$ position indique le sujet de la $3^{\mathrm{e}} \mathrm{p}$. (sing. zéro, plur. hä-, indéfini $t^{\prime} a^{-}$-). Les préfixes objectifs apparaissent dans la $6^{\mathrm{e}}$ position (sing. 1 ère p. sä-, $2^{\mathrm{e}}$ p. nä-, $3^{\mathrm{e}} \mathrm{p}$.

$\left.{ }^{16}\right)$ Delaware, an Eastern Algonquian Language by C. F. Voegelin, H., p. $130-157$.

17) Chipewyan by Li Fang-Kuei, H., p. 398-423. 
yä-, plur. 1 ère p. et $2^{\mathrm{e}}$ p, nuhä-, $3^{\mathrm{e}}$ p. yä-, indéfini ?ä-). Les préfixes de la $9^{\mathrm{e}}$ position marquent le sujet à la tère p. (sing. $s$-, plur. $i^{-}$) et à la $2 \mathrm{e}$ p. (sing. nä- ou $n^{-}$, plur. $n$-). p. ex, yidq-yáa$n i-t-t i$ she has brought him in again' (yidda 'in', -na- preftixe itératif conservé dans la nasalisation de la voyelle $q$ en $y i d q-$, -yä-préfixe objectif de la $3^{\mathrm{e}} \mathrm{p} .,-n i-$ préfixe momentané de la $3^{\mathrm{e}} \mathrm{p}$. du parfait, $-t$ - préfixe classificateur, $-t i$ to handle a living being racine; $H$. 418). Les préfixes objectifs du prédicat ont la même forme que les préfixes possessifs du nom.

Le sujet et lobjet nominaux fonctionnent comme appositions des préfixes pronominaux du prédicat et ne se distinguent que par la position de l'objet avant le prédicat et après le sujet: $(S)+(O)+(s-o-P)$. La proposition intransitive ne diffère de la transitive que par l'absence de l'objet pronominal et nominal: $(S)+(s-P)$. L'objet indirect est placé immédiatement après le sujet: $(S)+\left(O^{\prime}\right)+(O)+(s-o-P)$, p. ex. sä-tîua sa kún ७ä-t-tsị 'my grandson made a fire for me' (sä- 'my', -tîuä grandson'; sa for me'; kún fire'; ७ä- parfait, - $t$ - préfixe classificateur, -tsi to make' racine; la forme analysée ne contient pas le préfixe objectif -yä-, car si l'objet nominal précède immédiatement le prédicat, ce préfixe est souvent omis; H. 413,415, 422). Le groupe nominal forme un composé dans lequel le premier membre désigne le possesseur et le second, élargi souvent par le suffixe possessif -áa, indique la chose possédée: $(P r-P e-a ̈)$, p. ex. däne-bán-á 'a war party of Indians' (däne 'person, Indian', bán-á 'war party', H. 402).

La même structure de la phrase, concentrique du deuxième degré et analytique, caractérise le tlingit $\left.{ }^{18}\right)$, langue parlée dans le Sud-Est de l'Alaska à commencer par la Copper river jusqua Portland canal qui, ainsi que le groupe athapaskan, appartient à la famille na-dene. Le prédicat placé à la fin de la phrase contient des préfixes indiquant le mode, l'aspect et la personne de l'objet et du sujet. Le préfixe objectif apparaît à la tête du groupe prédicatif, c.-à-d. dans la première position: sing. $1^{\text {ère }}$ p. $x A t^{-}, 2^{\mathrm{e}} \mathrm{p}, i^{-}, 3^{\mathrm{e}}$ p. $a^{-}, d u^{-}$ou

${ }^{18)}$ Tlingit, by John R. Swanton, B., p. 159-204. 
Ac-; plur. $1_{\text {ère }}$ p. $h a, 2^{\mathrm{e}}$ p. $y^{\bar{\imath}}, \overline{3}^{\mathrm{e}}$ p. $a^{-}, h A s-$ ou $h A s d u^{-}$. Le sujet est indiqué par le préfixe pronominal placé dans la $4^{\mathrm{e}}$ position: sing. $1^{\mathrm{e} r e}$ p. $x$ - ou $x a^{-}, 2^{\mathrm{e}}$ p. $i^{-}, 3^{\mathrm{e}}$ p. zéro; plur. 1 ère p. tu-, $2^{\mathrm{e}}$ p. yi-, $3^{\mathrm{e}}$ p. zéro. Les préfixes objectifs du prédicat ne diffèrent des préfixes possessif́s du nom que dans la tère p. (objectif $x A t-:$ possessif $A x-$ ). Le prédicat transitif dérivé de la racine transitive contient le préfixe objectif et subjectif: (o-s-P.tr. , p. ex. iq! Axawiststin T questioned thee (i-q!A-xa-wü-s! în, i- thee' objet pronominal, q!A- mouth' préfixe nominal, $x a-$ '\% préfixe subjectif, wu- prêfixe verbal, -stîn to question' racine, $B .171$ ); $i-x a-s i-t i^{-1} n$ I saw thee' ( $i$ - 'thee' $x a^{-}$'I', si-pré-

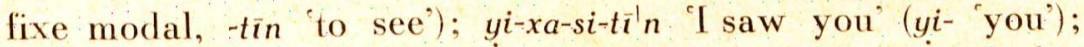
$x A t-y i-s i-t i{ }^{\prime} n$ 'ye saw me' (xAt- 'me', yi- 'ye'); ha-yi-si-tīn 'ye saw us' ( $h a-$ 'us'); $h A s-y i-s i-t \iota^{\prime} n$ ye saw them' ( $h A s-$ them', $B$ 171).

Le sujet nominal et l'objet nominal caractérisés uniquement par leur place dans la proposition fonctionnent comme appositions des préfixes pronominaux du prédicat: $(S)+(O)$ $+(o-s-P . t r$.$) , p. ex dusî qok!tit! ak { }^{\prime \prime} c \hat{t} A^{\prime} n$ his daughter liked to pick berries' (du- his', sī daughter' sujet; qok!tit! 'berries' objet; $a-k^{u}-c \hat{\imath}-t A^{\prime} n$ prédicat, $a^{-}$objet pronominal de la $3^{\mathrm{e}} \mathrm{p} ., k^{u-}$ préfixe modal, $c \hat{\imath}$ - préfixe modal de valeur optative, $t A n$ to pick; le sujet pronominal de la $3^{\mathrm{e}} \mathrm{p}$. apparaît sous la forme zéro; $B$ 184).

Le prédicat intransitif dérivé d'une racine intransitive ne contient que le préfixe subjectif indiquant le sujet: $(s-P)$, p. ex. xagāx I am crying' ( $x a-$ - l'préfixe subjectif, -gax 'to cry', B. 171). La proposition intransitive est construite d'après le schème: $(S)+(s-P)$, p. ex. Kîks $A^{\prime}$ dî qot cü'waxix the Kiksadi were all lost' (Kîks A'dî sujet; qot 'wholly'; cū'-wa-xix prédicat, cu- totally', $w a-$ préfixe verbal indiquant l'aspect perfectif, -xix to finish', préfixe subjectif zéro; $B$. 174); uhă'n gAx tu'satîl 'we are crying' (uhă'n 'we pronom indépendant dans la fonction du sujet; $g A x$ to cry'; tu- 'we' préfixe subjectif du prédicat, sa- préfixe verbal signifiant état, $-t i$ to be'; $B$. 171).

L'objet indirect de la $3^{\mathrm{e}} \mathrm{p}$. est indiqué par le préfixe $d a^{-}$, p. ex. dukā'niyên ye daya duqa his brothers-in-law spoke to him thus' (du- 'his ', kä'niyên 'brothers-in-law' sujet; ye 'thus'; da-ỵa'-du- 
$q a$ prédicat, da- 'to him' objet indirect, ya- préfixe duratif, du- préfixe perfectif, -qa to say'; $B$. 174).

Le groupe nominal apparaît sous la forme d'un composé où le premier membre indique le possesseur et le second la chose possédee: $(P r-P e), p$. ex. gAgāln-q!òs 'sun-feet' (=sunbeams), gAgā'n 'sun', q!ōs 'feet' ( $\dot{B} .16 z)$.

$\$ 13$. Passons maintenant à l'aztèque ${ }^{19}$ ), langue concentrique du troisième degré qui ne distingue pas formellement le sujet et l'objet nominaux, langue parlée en Mexique par un million d'individus. Il y a en aztèque une opposition entre le prédicat verbal qui est conjugué et le prédicat nominal qui n'est pas fléchi et qui est composé d'un pronom indépendant et d'un nom (substantif, adjectif ou adverbe), p. ex, hwa'n

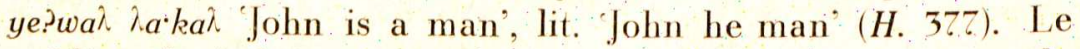
prédicat verbal caractérisé par les suffixes de l'aspect et de la voix et par les préfixes pronominaux apparaît sous quatre formes.

$1^{\circ}$ Le prédicat intransitif ne contient que le préfixe subjectif: sing. 1 ère p. ni-, $2^{\mathrm{e}}$ p. ti-, $3^{\mathrm{e}}$ p. zéro; plur 1ère p. $t i-, 2^{\mathrm{e}} \mathrm{p}$. $a m-, 3^{e}$ p. zéro. Dans cette fonction peut apparâttre un verbe intransitif ou un verbe transitif dans la forme intransitive, p. ex. hapan-tok lies broken' (suffixe -tok forme un intransitif du verbe transitif -hapana 'break' H. 387). Le sujet nominal n'est que l'apposition du prédicat intransitif: $(s-P)-(S)$.

$2^{\circ}$ Le verbe transitif dans la fonction du prédicat transitif contient un préfixe subjectif et après lui un préfixe objectif ; sing. 1 ère p. ne $\check{c}^{-}, 2^{\mathrm{e}}$ p. mi $c^{-}, 3^{\mathrm{e}}$ p. ki-; plur. 1 ère p. te $\breve{c}^{-}$, $2^{\text {e }}$ p. ame $\check{c}_{-}, 3^{\text {e }}$ p. kim-. Dans la proposition transitive: $(s-o-P)-$ (S) - (O) l'objet nominal - de même que le sujet - fonctionne comme apposition du préfixe objectif du prédicat, p. ex. kiči wa kalli he makes the bouse' (préfixe subjectif de la $5^{\mathrm{e}}$ p. zéro, ki- préfixe objectif de la $3^{\mathrm{e}} \mathrm{p}$., či $w a$ to make; kalli 'house' objet, apposition du préfixe objectif ki-; $H$. 384). Le sujet et lobjet nominaux ne se distinguent ni par la forme ni par la position, mais seulement par le sens général de la

${ }^{19}$ ) The Milpa Alta Dialect of Aztec by Benjamin Le Whorf, H. 367-379. 
phrase ou par lincorporation de l'objet dans la forme du prédicat. Dans ce dernier cas on obtient une proposition intransitive du type: $(s-O-P)-(S)$, dans laquelle l'objet nominal $(O)$ se présente formellement comme membre déterminant d'un composé verbal, p. ex. me+pam-po?powa 'weeds agave-row(s)', préfixe subjectif de la $3^{\mathrm{e}} \mathrm{p}$. zéro, me- $\lambda$ 'agave' pami- $\lambda$ 'row', po?powa weed' (H. 378). La fonction syntaxique du prédicat est augmentée encore par des préfixes comparables aux français $y$ et en qui indiquent la direction de l'action par rapport au sujet et à l'objet, p. ex. k-on-ana onward-gets it, goes and gets it' (préfixe subjectif zéro et objectif $k$ - de $k i$-, préfixe de la direction centrifugale on-, ana 'get', H. 384).

$5^{\circ}$ Le prédicat causatif dérivé du verbe transitif au moyen du suffixe-ltia contient trois préfixes pronominaux. Le premier préfixe, subjectif (abr. s), exprime le sujet de l'action causative, c.à-d. de cette action qui cause une autre. Le deuxième préfixe, objectif (o), indique l'objet de l'action causative et en même temps le sujet de l'action causée par cette action. Le troisième préfixe, formellement aussi objectif $\left(\dot{o}^{\prime}\right)$, ne se distingue du deuxième que par sa position après lui. Il exprime l'objet de l'action causée par la première action. Ainsi le prédicat causatif est construit d'après lé schème suivant:

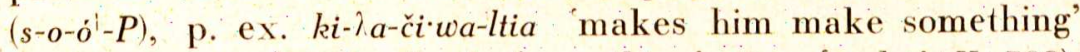
( $s=z e ́ r o, o=k i$ 'him', $\dot{o}^{\prime}=\lambda_{a}$ 'something', či $i w a$ 'make', H. 388). Les préfixes pronominaux du prédicat causatif peuvent obtenir des appositions nominales dont la fonction est précisée par le contexte.

$4^{\circ}$ Le prédicat à deux objets, direct et indirect, est dérivé du verbe transitif a moyen du suffixe appellatif et de trois préfixes pronominaux, c.-ì-d, le préfixe subjectif $(s)$, le premier préfixe objectif $\left(o^{\prime}\right)$ exprimant l'objet indirect et le deuxième préfixe objectif (o) qui indique l'objet direct: $(s-o \mid$ -o-P), p. ex. ki-la-čiwa-lia makes something for him' ( $s=z$ éro, $o^{\prime}=k i^{-}$- for him', $o=\lambda a$ 'something', či wa 'make', -lia suffixe appellatif, $H .388$ ). On voit que si dans le prédicat apparaissent deux préfixes objectifs, leur fonction est précisée par le suffixe causatif ou appellatif. 
Dans le groupe nominal le nom de la chose possédée $(P e)$ contient un préfixe pronominal possessif (pos.) qui diffère du pronom objectif. Le nom du possesseur $(P r)$ fait apposition de ce préfixe: $(P r)-($ pos. $P e)$, p. ex. kone $\lambda_{i}$-kal the child's house', lit. 'the child his house' (kone $\lambda$ child', $i$ - his', kal 'house', H. 381).

$\S 14$. La proposition dans la langue apache ${ }^{27}$ ) du groupe athapaskan parlée au Nouveau Mexique et en Arizona possède une structure concentrique intermédiaire entre le troisième et le quatrième degré de concentration syntaxique. Le prédicat caractérisé par les préfixes pronominaux et par les préfixes de l'aspect, du mode et de la voix apparaît en apache sous trois formes principales:

$1^{\circ}$ Le prédicat intransitif dérivé de la racine verbale transitive ou intransitive ne contient que des préfixes subjectifs indiquant le sujet: $(s-P)$. Voici les préfixes subjectifs du singulier: 1 ère p. $\check{s}^{-}, 2^{\mathrm{e}}$ p. $\grave{n}-, 3^{\mathrm{e}}$ p. $\breve{z} i$ - (préfixe honorifique indiquant un certain groupe de parents), go- (, a place or time subject", H. 79), ? $i$ - (sujet indéfini), zéro (dans tous les autres cas), p. ex. hišbé.ž I boil' (hi-š-bé. $z$, hi- préfixe prédicatif, -š- I', -bé. $\check{z}$ to boil'); 'n-bé.ž you boil (sing.)'; hi-bés the boils' (sujet indiqué par le préfixe zéro), žzi-béšs he boils' (préfixe honorifique de la $3^{\text {e }}$ p. $\mathfrak{z}^{-}$-). Le sujet nominal peut apparaître comme apposition du préfixe subjectif du prédicat: $(S)-(s-P)$.

$2^{\circ}$ Le prédicat transitif dérivế de la racine verbale transitive contient deux préfixes pronominaux, objectif et subjectif: $(o-s-P)$. La série objective identique à la série des préfixes. possessifs des noms est composée au singulier de préfixes suivants: 1 ère p. ši-, $2^{\mathrm{e}} \mathrm{p}, n i-, 3^{\mathrm{e}} \mathrm{p}$. go- (préfixe honorifique), yi(préfixe employé dans les autres cas), p. ex. ništbé.ž ( $\leq n i-s ̌-l$ bé.ž) 'I boil you' (ni- you' préfixe objectif, -š- T' préfixe subjectif, - $t$ - classificateur indiquant la voix, bé:ž to boil'; $H . \quad 79)$; $y \dot{i}-t-b \dot{e} \cdot z$ 'he boils it' (yi- it' objet; le sujet est indiqué par le préfixe zéro; $H .82$ ). Dans la proposition transitive le sujet et l'objet nominaux ne sont pas différenciés par la forme, mais

$\left.{ }^{20}\right)$ Chiricahua Apache by Harry Hoijer, H., p. 55-84. 
l'existence de plusieurs préfixes subjectifs et objectifs de la $3^{\mathrm{e}} \mathrm{p}$. permet en un certain degré de déterminer la fonction des membres nominaux de la phrase. Grâce à ce fait on peut considérer l'apache comme langue qui s'approche du quatrième degré de la concentration syntaxique.

$3^{\circ}$ Le prédicat transitif à deux objets, indirect (o $o^{\prime}$ et direct $(o)$, possède la structure suivante: $\left(o^{\prime}=0-s-P\right)$. Si le sujet est à la lère p. ou à la $2^{\mathrm{e}} \mathrm{p}$., l'objet indirect à la $5^{\mathrm{e}} \mathrm{p}$. est indiqué par un préfixe spécial bi-. Dans tous les autres cas l'objet indirect est exprimé par des préfixes objectifs, les mêmes qui sont employés pour l'objet direct. Les objets pronominaux ne sont distingués que par la position de lobjet indirect avant l'objet direct. Ainsi dans l'opposition préfixe subjectif : préfixe objectif c'est le premier morphème qui a la fonction d'un terme positif et le deuxième d'un terme négatif.

Le groupe nominal possède en apache la forme diun composé, dans lequel le premier membre indique le possesseur et le second la chose possédée: $(\mathrm{Pr}-\mathrm{Pe}), \mathrm{p}$. ex. ci-tóa top of the head' (ci-- head' -t'a 'top', H. 75).

Le système des oppositions des préfixes prédicatifs destinés à distinguer le sujet et l'objet nominaux, le système existant en état embryonnaire en apache, a pris une forme plus développée en hupa ${ }^{21}$, un autre dialecte du groupe athapaskan parlé en Californie septentrionale. Le prédicat intransitif $(s-P)$ contient en hupa des préfixes subjectifs suivants: sing. 1ère p. $w-, 2^{\mathrm{e}}$ p. $\dot{n}^{-}-3^{\mathrm{e}}$ p. la classe A. supérieure $t c-$, la classe B. inférieure $y^{-}$; plur lère p. $d^{-}, 2^{\mathrm{e}} \bar{o}^{-}, 3^{\mathrm{e}} \mathrm{p}$. A. $y a^{-}$, $B$. yay-. Ainsi il y a en hupa une opposition de genre exprimée par des préfixes subjectifs de la $3^{\mathrm{e}} \mathrm{p}$. Des hommes et des femmes adultes de la tribu Hupa appartiennent à la classe supérieure, et la classe inférieure contient tout le reste de phénomènes (êtres vivants et choses inanimées), p. ex. ye-tc-iLda he (an adult Hupa) is carrying a large object: $y e-y-i L d a$ he (not an adult Hupa) is carrying a large object' (ye- préfixe adverbial signifiant a large object, $-t c^{-}:-y^{-}$l'opposition du

${ }^{21}$ ) Athapascan (Hupa), by Pliny Earle Goddard, B., p. 85-158. 
genre des préfixes de la $3^{\text {e }}$ p.; $B$. 117). Dans la proposition intransitive $(S)-(s-P)$ le préfixe subjectif du prédicat s'accorde en genre avec le sujet ce qui permet de reconnaître sa fonction syntaxique, p. ex. Médildin dedin tcitteLtcwen lit. Medildiń poor he grew' (Me dildin' 'dans Medildiń' adverbe local tiré d'un nom de lieu; dedin poor, not having possessions' sujet nominal; tci-tte-L-tcwen he grew' prédicat, tci- he' le préfixe subjectif de la $3^{\mathrm{e}} \mathrm{p}$. sing. de la classe supérieure qui s'accorde avec la classe du sujet désignant un adulte Hupa, -te- préfixe local, - $L$ - préfixe modal, -tcwen to grow'; $B$. 153).

Les prédicats transitifs contiennent des préfixes subjectifs et objectifs: $(s-o-P)$. La série objective identique aux préfixes possessifs des noms n'admet pas la distinction en genre dans la $3^{\mathrm{e}} \mathrm{p}$. Voici les préfixes objectifs du hupa: sing. 1ère p. $h w-, 2^{\mathrm{e}}$ p. $n^{-}, 3^{\mathrm{e}}$ p. $x \bar{o}$; plur. $1^{\mathrm{e}} \mathrm{e}$ p. et $2^{\mathrm{e}} \mathrm{p}$ nō-, $5^{\mathrm{e}}$ p. yaxó-, p. ex. $\left.t c(\bar{u})-h w-\bar{o}-w^{\prime} i\right)-L-x \hat{u} l(i)-L-t e$ 'she will ask me for it' (tc-'she' sujet pronominal à la $5^{\mathrm{e}} \mathrm{p}$. de la classe supérieure, - $h w$ - me' objet pronominal, $-o^{-},-w$ - et $-L$ - les préfixes modaux, $x \hat{u} l$ to ask', - $L$ - continuatif, -te futur); ta-na-i-xō-s-dōw-ei it cut him all to pieces' (ta- préfixe adverbial, na- préfixe itératif, $i^{-}=-y^{-}$ it' sujet pronominal a la $3^{\mathrm{e}} \mathrm{p}$. de la classe inférieure, $-x \bar{o}-$ him' objet a la $3^{\mathrm{e}} \mathrm{p} .,-s-$ préfixe modal, -dō- to cut racine, -ei suffixe emphatique; $B$ 118).

Dans la proposition transitive du type $(S)-(s-o-P)-(O)$ ou $(S)-(O)-(s-o-P)$ c'est l'ordre des mots qui permet de distinguer le sujet placé à la tête de la phrase de l'objet, si tous les deux sont exprimés par des noms du même genre, mais lorsque les membres nominaux de la proposition sont du genre différent ou le prédicat transitif n'est accompagné que d'un nom, la fonction syntaxique des noms est précisée par l'accord ou par le manque d'accord avec le préfixe subjectif du prédicat. Dans le premier cas le nom fonctionne comme sujet, mais le manque d'accord en genre et en nombre avec le préfixe le caractérise comme objet, p. ex. haiûn tak tceseLwen mikkyaqōttse lit. 'then three he killed elks' (haiûn then'; tak 'three'; tce- he préfixe subjectif de la $3^{\mathrm{e}} \mathrm{p}$. de la classe supérieure, -se- et $-L$ - préfixes modaux, wen to kill racine; mikkyaqōttse 
'elks' le nom de la classe inférieure au pluriel qui, par le manque d'accord avec le préfixe tce- du prédicat, se caractérise comme objet; $B .155-6)$. Le sujet nominal constitue l'apposition du préfixe subjectif du prédicat qui, de son côté, est souvent lié à l'objet par laccord quant à la classe indiquée par sa racine. En hupa comme en haida la classificition est faite d'après la forme extérieure des objets. Ainsi certaines racines verbales indiquent des objets longs, ronds, plats, des êtres animés etc. Dans la proposition transitive, la classe de la racine du prédicat s'accorde avec la classe de l'objet grammatical, p. ex. medil taïntüw lit. 'canoe take out of the water' (medil canoe' objet; ta- 'out of the water' préfixe adverbial, -inpréfixe subjectif de la $2^{\mathrm{e}} \mathrm{p}$., -tūw la racine verbale qui n'est employée que pour les objets longs et qui par sa classe s'accorde avec l'objet grammatical, c.-a-d. un canot; B. 158). Dans les propositions intransitives: $(S)-(s-P)$ la classe de la racine du prédicat indique le sujet, et cette analogie dans la construction de l'objet et du sujet intransitif approche le hupa des langues subjectives.

Dans les groupes nominaux du type: $(P r)-($ pos $-P e)$ le nom du possesseur $(P r)$ fonctionne comme apposition du préfixe possessif (pos.) précédant le nom de la chose possédée, p. ex. taikyüw mittsitda' lit. 'sweat-house its roof' (taikyūw 'sweathouse'; mit- préfixe possessif, -tsitda' 'roof'; B. 158).

$\$ 15$. Passons maintenant aux langues concentriques et en même temps objectives comme p. ex. eskimo ${ }^{22}$ ). Dans les dialectes eskimos parlés sur les côtes du Groenland il y a deux séries de suffixes pronominaux: la série objective (abr. o, sing. $1^{\text {ere }}$ p. - - $a, 2^{\text {e }}$ p. -tit, $3^{\text {e }}$ p. $-q$; plur. $1^{\text {ere }}$ p. - aut, $2^{\text {e }}$ p. -se, $5^{\mathrm{e}}$ p. $\left.-t\right)$ et la série subjective-objective (abr. so, p. ex. rma tu me...', -anit je te... etc.). Le système morphologique de ces dialectes est basé sur l'opposition des trois parties du discours, c.-̀̀-d.

${ }^{22}$ ) Cf. Eskimo, by William Thalbitzer, B., p. C67-1669; South Greenlandic (Eskimo) by Morris Swadesh, H., p. 30-54; Michella Erichsen, Délandic (Eskimo) by Morris Swadesh, H., p. $30-$ Acta Linguistica IV, p. 67-88;
sinences casuelles et personnelles en eskimo, Acta de cas, Deuxième partie. Acta Jutlandica
Louis Hjelmsler, La catégorie des cos, IX, 2, Kobenhavn 1937, p. 65-75, 
des verbes transitifs, des verbes intransitifs et des noms. Les verbes transitifs caractérisés par des suffixes du mode et du temps peuvent apparaître sous la forme du prédicat intransitif contenant un suffixe objectif $(P-o)$, p. ex. kapiwo-ra I stab myself" ou dans la forme du prédicat transitif élargi par un suffixe subjectif-objectif $(P$-so $), p$. ex. kapiwa-anit I stab thee'. Les verbes intransitifs n'apparaissent qu'en fonction du prédicat intransitif contenant un suffixe objectif $(P-o)$.

Les noms sont fléchis au moyen des désinences casuelles et des suffixes objectifs qui ont dans ce cas la fonction des pronoms possessifs, p. ex. i $\gamma$ lo-ra my house en absolutif: $i \vee l u-m-a$ my house' en relatif. Dans la déclinaison des noms il y a deux formes purement syntaxiques: l'absolutif à la finale zéro, p. ex. iүlu a house, the house', et le relatif en -p, p. ex. iүlu-p. Ces deux formes indiquent que le nom apparaît dans la fonction d'une apposition du suffixe pronominal d'un verbe ou d'un nom. Dans l'opposition relatif: absolutif c'est le relatif en $-p$ qui fonctionne comme terme positif. Il indique que le nom fait apposition du suffixe subjectif-objectif d'un verbe ayant la valeur d'un agent $(A)$ ou du suffixe objectif (possessif) d'un nom en fonction du possesseur. L'absolutif à finale zéro est morphologiquement et syntaxiquement le terme négatif de l'opposition. Il est employé dans tous les autres cas, c.-à-d. comme objet transitif faisant apposition du suffixe subjectif-objectif d'un prédicat transitif et comme objet intransitif (le sujet intransitif des langues indo-européennes) qui détermine le suffixe objectif d'un prédicat intransitif. En eskimo la proposition transitive possède la structure suivante:

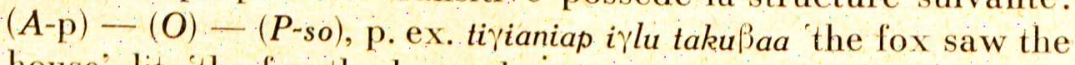
house, lit. 'the fox the house he saw it'.(tiyia-nia-p the fox' agent

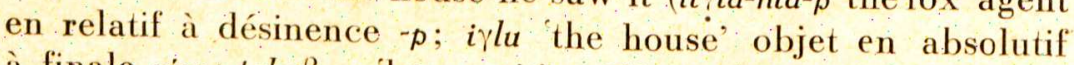
à finale zéro; takußa-a he saw it' prédicat contenant le suffixe subjectif-objectif $-a$ il le $\ldots, H, 45)$. Le centre de la proposition se trouve dans le prédicat à désinence subjective objective il le..,' et les membres nominaux de la phrase différenciés par leurs finales fonctionnent comme appositions de cette désinence. 
La proposition intransitive est composée de l'objet intransitif en absolutif et du prédicat à désinence objective: $(O)-(P-o)$,

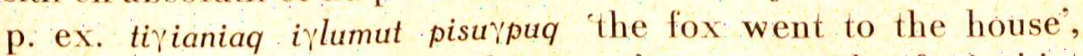
lit. 'the fox to the house he went' (tivianiaq 'the fox' objet intransitif en absolutif; irlu-m-ut to the house' allatif a finale -ut, pisurpu q he went' prédicat à désinence objective de la $3^{\mathrm{e}}$ p. $\left.-q\right)$.

Dans le groupe nominal le nom du possesseur élargi par la désinence $-p$ du relatif fait apposition du suffixe objectifpossessif du nom de la chose possédée: $(\operatorname{Pr}-\mathrm{p})-\left(P_{e-o}\right)$, p. ex.

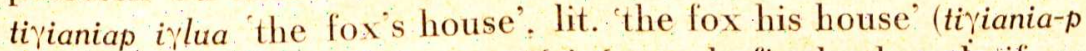
'the fox', le possesseur caractérisé par la finale du relatif $-p$; iүlu-a his house, - $a$ suffixe objectif-possessif de la $3^{\mathrm{e}}$ p.; $H .45$ ).

Le système d'appositions nominales en -p et en zéro de l'eskimo est assez proche du système d'appositions y uma en -c et en zéro (cf. \$10), mais à côté de lui on trouve en eskimo un système casuel très éloigné du type normal des langues de l'Amérique du Nord. En eskimo il y a six formes locales, c.-à-d. l'ablatif en -nit from', l'allatif en -nut into', le prosécutif en-rut 'through', le locatif en -ne 'in', l'équatif en -sut like' et l'instrumental en -nik 'by'. Parmi ces formes l'allatif en -nut au moins doit être considéré comme le vrai cas à-double fonction locale et syntaxique. La première fonction consiste dans l'indication du point final d'un mouvement. En même temps l'allatif a trois fonctions syntaxiques. Il peut exprimer le sujet logique d'un verbe impersonnel: $(S-n u t)-(P-o), \mathrm{p}$. ex. taman-nut takunarpu-q it was seen by all', lit. 'to all one saw it'. Dans la proposition causative l'allatif désigne l'objet de l'action causante, c.-a-d. de cette action qui cause une autre, et il constitue en même temps le sujet de l'action causée par la première action: $(S)-(O$-nut $)-(P-s), p$. ex. pini inus-nut takuүqußa-i he wants people to see his things', lit. 'his possessions - to people - he wants one te see them'. Enfin l'allatif possède la fonction d'un objet indirect: $(O)-\left(O^{\prime}\right.$-nut $)-(P$-so $)$, p. ex. anirausat inur-nut tuniuppa-i he gave the money to the people', lit. 'the money (plur.) - to the people - he gave them? (H. 46-47). 
$\S$ 16. En chitimacha ${ }^{23}$ ), langue du groupe tunica parlée jadis dans le Sud de la Louisiane, la proposition possède la structure concentrique du deuxième degré, objective et en même temps synthétique. Il y a en chitimacha trois parties du discours: le verbe, le substantif et l'adjectif. Les verbes ayant la fonction d'un prédicat verbal sont fléchis d'après des paradigmes compliqués. Les substantifs (y compris les pronoms) ne sont pas fléchis et apparaissent dans la fonction du prédicat nominal avec un verbe auxiliaire ou dans la fonction d'un autre membre de la phrase. Les adjectifs enfin peuvent apparaître sous la forme et dans la fonction des substantifs ou des verbes. Le prédicat est caractérisé par sa position à la fin de la proposition et par les désinences personelles du verbe, de l'auxiliaire ou de l'adjectif, Ces désinences ont créé un système à deux termes où la lère p. s'oppose à la forme commune de la $2^{\mathrm{e}}$ et $3^{\mathrm{e}} \mathrm{p}$. En chitimacha il y a quatre
types de prédicats:

$1^{\circ}$ Dans le prédicat transitif dérivé d'un verbe transitif les désinences personelles indiquent en même temps l'objet transitif $(o)$ à la 1 ère p., $2^{\mathrm{e}} \mathrm{p}$. ou $3^{\mathrm{e}} \mathrm{p}$. et l'agent $(a)$ à la $3^{\mathrm{e}}$ p.: $(P$-oa $)$, p. ex. getki (de la racine get-) the beat me', geti he beat thee' ou 'he beat him' $(H \cdot 325)$. Ainsi la désinence -ki//-k indique l'agent à la $3^{\mathrm{e}} \mathrm{p}$. et l'objet transitif à la lère p. pendant que la finale $-i$ désigne l'agent à la $3^{\mathrm{e}} \mathrm{p}$. et l'objet à la $2^{\mathrm{e}}$ ou $3^{\mathrm{e}} \mathrm{p}$. L'agent nominal $(A)$ et l'objet nominal $(O)$ ne se distinguent que par leur place dans la proposition et fonctionnent comme appositions des désinences personnelles du prédicat: $(A)+(O)+(P-o a)$, p. ex. we panšl kišs getỉi 'that man killed a dog', lit. 'that (we) man (panšl) $\operatorname{dog}\left(k i s^{\prime}\right)$ thither (hi) he-killed-him (geti.ji, H. 31z). Cette construction caractérise le chitimacha comme langue
concentrique du deuxième degré.

$2^{\circ}$ Dans la fonction d'un prédicat intransitif peut apparaître un verbe intransitif, un adjectif fléchi ou un substantif suivi d'un verbe auxiliaire. Dans tous ces cas les désinences personnelles indiquent l'objet intransitif correspondant au sujet

$\left.{ }^{23}\right)$ Chitimacha by Morris Swadesh, H., p. 312-336. 
intransitif des langues subjectives: $(P-o)$, p. ex. hik 'I am', $h i$ 'you are' ou 'he is'. Ainsi en chitimacha chaque désinence personnelle indique toujours l'objet transitif ou intransitif de la même personne. En plus, dans les verbes transitifs elle indique l'agent à la $3^{e} \mathrm{p}$. La désinence $-k i / /-k$ qui indique dans les verbes transitifs lagent à la $5^{\mathrm{e}} \mathrm{p}$. et lobjet transitif à la lère p.: ‘il me.. $(o a)$, indique dans le prédicat intransitif l'objet intransitif à la lère p.: 'je..' (o). Ainsi le chitimacha est une langue synthétique, car il n’y a qu une série indifférenciée de désinences personnelles qui dans le prédicat transitif expriment l'agent et l'objet transitif et dans le prédicat intransitif l'objet intransitif tout seul. En même temps le chitimacha est une langue objective, car la même désinence indique dans notre cas lobjet transitif et intransitif.

$3^{\circ}$ Dans les verbes causatifs les mêmes désinences personnelles indiquent l'objet de l'action causant une autre action et en même temps le sujet de l'action causée par la première action, p. ex. tučpaki he made me cook it' (H. 326). De la racine tuč- 'to cook' on a dérivé au moyen du suffixe -pa- la forme causative tučpa- élargie par la désinence de la $1^{\text {ère }} \mathrm{p}$. - $k i$ qui indique l'agent de l'action causant une autre action à la $3^{\mathrm{e}}$ p. (he), l'objet de cette action et le sujet de l'action causée en 1 ère p. (me) et enfin l'objet de l'action causée à la $3^{\mathrm{e}} \mathrm{p}$. (it). $4^{\circ} \mathrm{Si}$ le verbe apparaît en indiréctif, la désinence personnelle indique l'objet indirect, p. ex. tuča.ki 'he cooked it for me' (H. 326). La désinence de la 1ère p. -ki indique dans ce cas l'agent (he) et l'objet direct (it) à la $3^{\mathrm{e}}$ p. et l'objet indirect (me) à la 1ère p. Ainsi la valeur des désinences personnelles en chitimacha dépend de la structure morphologique du verbe. Dans le groupe nominal le possesseur n'est caractérisé que par sa position avant le nom de la chose possédée $(P r)+(P e)$, p. ex. we ?asi ?inž $i$ that man's father', lit. 'that (we) man (?asi) father (?inži, H: 333 )'.

$\$ 17$. Le dakota ${ }^{24}$ ) du groupe siou parlé jadis dans l'État Dakota appartient au type des langues concentriques du troi-

24) Siouan, Dakota (Teton and Santee dialects) with remarks on the

Ponca and Winnebago, by Franz Boas and John R. Swanton, B., p. 875-965. 
sième degré, objectives et en même temps analytiques. En dakota il y a trois séries de préfixes pronominaux, c-à-d. la série subjective indiquant l'agent (abr. $a$ : sing. 1ère p. wa-, $2^{\mathrm{e}} \mathrm{p}$. ya-, $3^{\mathrm{e}}$ p. zéro), la série objective qui désigne l'objet transitif et intransitif correspondant au sujet intransitif des langues indo-européennes (abr. o: sing. 1ëre p. ma-, $2^{\mathrm{e}} \mathrm{p} . n i^{-}, 3^{\mathrm{e}} \mathrm{p} . z e ́ r o$ ) et enfin la série subjective-objective indiquant en même temps l'agent et l'objet (abr. oa, p. ex. maya- tu me..., ci- je te...' etc.). Les préfixes de cette dernière série sont composés pour la plupart de préfixes des deux séries précédentes (p. ex. ma-ya- tu me ...'). Dans ce cas le préfixe objectif (ma- 'me') précède le préfixe subjectif (-ya- tu'). Au moyen de ces préfixes pronominaux on dérive en dakota les prédicats des quatre types différents:

$1^{\circ}$ Le prédicat transitif indiquant l'agent pronominal et l'objet est dérivé de la racine transitive au moyen des préfixes subjectifs-objectifs (oa-P.tr.), p. ex. maya'kte thou killest me' (maya- thou ... me', -kte to kill' B. 909).

$2^{\circ}$ Le prédicat transitif sans objet est composé du préfixe subjectif et de la racine active (a-P.tr.), p. ex. wa-t $i$ 'I dwell' (wa- préfixe subjectif de la 1 ère $p$, $t i$ he dwells, B. 909).

$3^{\circ}$ Le prédicat intransitif contient la racine intransitive élargie par le préfixe objectif indiquant l'objet intransitif (o-P.intr.), p. ex. ma-síca 'I am bad' (ma- préfixe objectif de la 1 ère p., B. 909).

$4^{\circ}$ L'objet pronominal indirect est indiqué au moyen du préfixe subjectif-objectif suivi du préfixe $k i-(c i-$ après $i)$, p. ex. ci-ći-dowq-pi I sing for you' (le premier ci- est le préfixe subjectif-objectif 'I ... you', le second ci-de ki-transforme la signification du premier préfixe en 'I ... for you', dowq- 'to sing', - $p i$ suffixe indiquant le pluriel de l'objet; $B .912$ ).

Le prédicat est caractérisé en dakota par sa position a la fin de la phrase, par des préfixes pronominaux et enfin par le suffixe $-p i$ indiquant que la proposition est au pluriel, p. ex. cap a tqk $a^{\prime}-p i$ 'large beavers' ou 'they are large beavers' (ća'pa beaver', tqka large', -pi pluriel, B. 932). Au contraire, 
les autres membres de la proposition: l'agent nominal et l'objet - qui peuvent être interprétés comme appositions du préfixe zéro du prédicat - ne sont caractérisés en dakota que par sa position avant le prédicat, p. ex. yu $q^{\prime}{ }^{\prime} q h e h q^{\prime} m A n i^{\prime}$ ekta' e'tqwq then he looked at the water' (yq'kq 'and then', hehq' 'then', mAni' water' objet transitif sans signe morphologique, ekta' 'at', e'-towq 'he looked' prédicat, -e pronom anaphorique; B. 957-8). L'objet nominal est souvent incorporé dans la forme du prédicat ce qui permet de le distinguer de l'agent, p. ex. ćq-ka'śka to tie wood together' (ćc-'wood', -ka'śka 'to tie', B. 893). Dans ce cas l'objet placé à la tête du groupe prédicatif fonctionne comme membre déterminant du composé.

§ 18. Passons maintenant aux langues concentriques du quatrième degré qui permettent de distinguer l'agent nominal de l'objet grâce à l'accord grammatical en genre et en nombre entre les affixes pronominaux de la $3^{e} \mathrm{p}$. du prédicat et les membres nominaux de la phrase. Une langue concentrique de ce type et en même temps une langue objective et synthétique est $\operatorname{taos}^{25}$ ) du groupe tano (la famille uto-aztèque) parlée dans le village (Pueble) Taos dans le Nord du NouveauMexique. Le prédicat caractérisé par des suffixes du temps et $\mathrm{du}$ mode contient en taos des préfixes pronominaux. Dans le prédicat transitif dérivé de la racine transitive ces préfixes indiquent en même temps l'agent et l'objet: (ao-P.tr.). Il y a deux séries principales de préfixes pronominaux, la série $C$ et la série $B$. La série $C$ contient douze préfixes indiquant l'objet dans la $1^{\text {ere }}$ p. ou dans la $2^{\text {e }}$ p. du singulier, du duel ou du pluriel et l'agent dans la $1^{\text {ere }}, 2^{\text {e }}$ ou $5^{\mathrm{e}} \mathrm{p}$. sans distinction des nombres. Voici les préfixes de la série $C$ :

\footnotetext{
L'agent

en sing.

et en pl. sing. 1 ere p. sing. $2^{\text {e }}$ p. duel tere $p$.

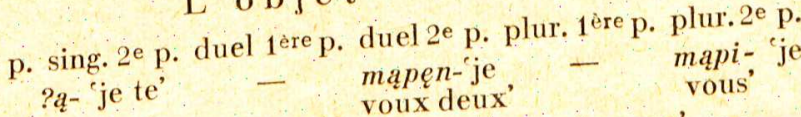

2e p. may-'tume - may- 'tu ${ }^{2}$ - mous deux

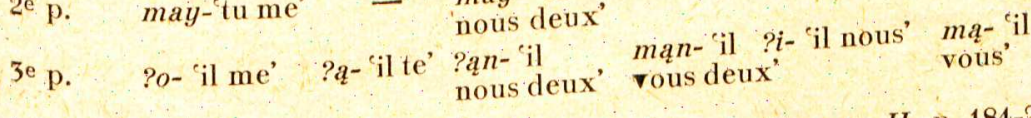

$\left.{ }^{25}\right)$ An outline of Taos grammar by George L. Trager, H., p. 184-221.
} 
Les préfixes de la série $B$ indiquant l'objet à la $3^{\mathrm{e}} \mathrm{p}$. opposent réciproquement trois classes d'objets: la classe $B \quad 1, B 2$ et $B$ 3. Cette opposition est basée sur le système des trois genres. Les noms animés appartiennent en taos au genre $I$, les noms inanimés au genre II et III sans répartition précise. Les préfixes prédicatifs de la classe $B /$ indiquent les objets du genre I et III au singulier, les préfixes de la classe $B 2$ les objets du genre II au singulier et du genre I au pluriel, enfin les préfixes de la classe $B 3$ les objets du genre II et III au pluriel. En plus on distingue à l'aide de la forme des préfixes de la série $B$ trois personnes et trois nombres (sing., duel, plur.) de l'agent. Ainsi il y a 27 préfixes de la série $B$ qui opposent trois classes d'objets $B 1, B 2, B 3$, trois personnes de l'agent et trois nombres de l'agent $(3 \cdot 5 \cdot 3=27)$. En ajoutant 12 préfixes de la série $C$ on obtient au total 39 préfixes subjectifs-objectifs du taos, p. ex. ti-c'lowq 'T tasted it' (ti- 'I ...it' préfixe subjectif-objectif de la classe $B l$ indiquant l'agent dans la $1^{\text {ere }}$ p. du sing. et l'objet du genre I ou III au sing.; c'owq to taste'; H. 208); ?o-mù-ya he saw me'( ?o- he...me' préfixe de la série $C$; $-m y^{-}$to see; -ya suffixe verbal; H. 209). En taos l'agent nominal et l'objet fonctionnent dans les propositions du type: $(A)-(O)-(a o-P . t r$.$) comme appositions des$ préfixes pronominaux du prédicat. La forme du préfixe indique la personne et le nombre de l'agent et la personne et la classe de l'objet ce qui permet de distinguer l'agent de l'objet, p. ex. timùhu ?quklana 'I see my mother', lit. 'I-her-see my-mother' (ti- 'I..her' préfixe indiquant l'agent dans la $1^{\text {ère }}$ p. et l'objet dans la $3^{\mathrm{e}} \mathrm{p}$. de la classe $B \quad 1$; ?qn- 'my', ?qn-klana my mother' apposition objective de la classe $B l$ au préfixe $t i-$ du prédi-
cat; H. 204).

Le prédicat intransitif dérivé de la racine intransitive ou de la racine transitive élargie par un suffixe intransitif, est conjugué au moyen de 15 prélixes indiquant trois personnes, trois nombres (singulier, duel et pluriel) et dans la $3^{\mathrm{e}}$ p. trois genres I, II et III. Ces préfixes, quoique formellement identiques aux préfixes désignant dans le prédicat transitif l'agent et l'objet transitif, n'indiquent dans le prédicat intransitif que 
l'objet intransitif. La personne et le nombre de cet objet intransitif sont identiques à la personne et le nombre de l'objet transitif désigné par ce préfixe dans le prédicat transitif. Ainsi les préfixes qui dans le prédicat transitif ont la valeur: ?o- 'il me...,? ?qn- il nous deux...,? $i^{-}$'il nous... (la série $C$ ) signifient dans le prédicat intransitif ?o je..., ?qn- 'nous deux...,? ?- nous..., p. ex. le prédicat transitif ?o-mùya he saw me', mais le prédicat intransitif ?o-cémamq I am young' (H. 209). Analogiquement les préfixes ?q-, mqn-, mq- (la série C) qui dans le prédicat transitif désignent il te...', il vous deux..., il vous...' signifient dans le prédicat intransitif 'tu...', vous deux..., vous...' et les préfixes zéro il le...,? qn- il les deux...? ? $i$ - il les..., (la série $B I$ ) indiquent dans le prédicat intransitif il ..., ils deux..., 'ils...' pour le genre I. Les préfixes $i_{i-}$ 'il le ...,? ?q- 'il les deux...,? ? $i^{-}$'il les...' (la série $B$ 2) fonctionnent dans le prédicat intransitif pour le genre II et les préfixes ? $u-$, ?qn-, ?iw- pour le genre III dans la valeur il ...', ils deux..., 'ils...'.

Le nombre et le genre du préfixe du prédicat intransitif s'accordent au nombre et au genre de l'objet intransitif (sujet logique) dans les propositions du type: $(O)-(a o-P$.intr. $), p$. ex. hux'u $y^{\prime}$ iat ${ }^{\prime}$ oti s'aonena wirapliwmę 'and the man went away from there sadly' (hux'u 'and' conjonction; $y^{\prime}$ iat ${ }^{c^{\prime}}$ oti 'there-from'; s'aonena 'man' objet intransitif; wỉapliwmé 'narrative-sad-went' prédicat avec le préfixe zéro qui indique l'objet intransitif dans la $3^{\mathrm{e}}$ p. du singulier du genre animé, à savoir 'man'; $H$ 215).

Ainsi le taos est une langue synthétique, car il n'a qu'une série indifférenciée de préfixes pronominaux qui dans le prédicat transitif expriment l'agent et l'objet transitif, et dans le prédicat intransitif l'objet intransitif tout seul. En même temps le taos est une langue objective, car le même préfixe indique dans son système l'objet transitif et intransitif.

La série $D$ de préfixes synthétiques du prédicat indique en taos l'agent et l'objet indirect: $\left(a o^{\prime}-P\right)$, p. ex. mqm- tu ...pour moi', kq- 'je...pour toi' etc. Si le préfixe désigne l'agent dans la $3^{\mathrm{e}} \mathrm{p}$. et l'objet indirect dans la $1^{\text {ère }}, 2^{\mathrm{e}}$ ou $3^{\mathrm{e}}$ 
p., l'objet direct est indiqué par le suffixe -ya: (ao'-P. tr.-o), p. ex. ?o-..-ya 'il le ... pour moi' (H. 210). L'objet indirect nominal constituant l'apposition du préfixe de la série $D$ s'accorde avec lui en personne, en nombre et en genre.

Dans le groupe nominal le nom de la chose possédée est précédé du préfixe possessif de la série $A 1, A 2$ ou $A 3$ parallèle et apparentée aux séries $B 1, B 2$ et $B 3$ des préfixes du prédicat transitif. Les préfixes possessifs indiquent le nombre (singulier et pluriel) de la chose possédée et la personne, le nombre (sing., duel et plur.) et le genre du possesseur. Dans le système de la flexion du taos le possesseur indiqué par le préfixe de la série $A$ correspond à l'agent désigné par les préfixes de la série $B$ du prédicat transitif et la chose possédée correspond à l'objet transitif. Le nom de la chose pussédée, précédé du préfixe possessif, est suivi en même temps du suffixe -wá? $i$ qui présente la forme du mode relatif du verbe auxiliaire wá- être', p. ex. ?q-puywá? $i$ his friend', lit. the one who is his friend' ? $q$ - his' préfixe possessif indiquant la chose possédée au singulier et le possesseur à la $3^{\mathrm{e}} \mathrm{p}$. du singulier du genre I, c.-̀-d. animé; -pluy-friend'; -wá? i auxiliaire; H. 206). Le nom du possesseur constitue l'apposition du préfixe possessif du nom de la chose possédée: $(P r)-($ pos.-Pe-wá? $i)$.

$\$ 19$. Les préfixes pronominaux en taos sont du type synthétique, car ils indiquent en même temps la personne, le nombre et le genre de l'agent et la personne et le nombre de l'objet. Au contraire, les affixes pronominaux du prédicat en tunica et en chinook possèdent la structure analytique. II y a dans ces langues des affixes subjectifs indiquant l'agent et des affixes objectifs indépendants qui déterminent l'objet. Les affixes de ces deux séries apparaissant dans le même prédicat forment la troisième série subjective-objective.

En tunica ${ }^{26}$ ) parlé jadis dans la partie du Nord de la Louisiane, la structure concentrique de la proposition est marquée par l'intonation. Le groupe syntaxique est composé

26) A grammatical sketch of Tunica by Mary R. Haas, H., p. 337-366. 
en tunica de plusieurs morphèmes délimités, par l'intonation de la dernière syllabe, du groupe suivant. L'intonation de la dernière syllabe du groupe prédicatif ne dépend que de son mode. Elle est haute en indicatif, basse en quotatif, ascendante en interrogatif et descendante en impératif. Au contraire, l'intonation de la dernière syllabe des autres membres de la phrase dépend de leur position par rapport au prédicat. Elle est ascendante dans les groupes syntaxiques placés avant le prédicat et descendante dans de rares groupes placés après lui. Ainsi l'intonation caractérise le prédicat comme une proposition complète exprimant son mode au moyen de l'intonation. Au contraire, l'intonation des autres membres de la phrase déterminée par la place du prédicat, caractérise leur dépendance à l'égard de ce membre principal.

En tunica, le prédicat, caractérisé par l'indépendance de l'intonation, peut être nominal ou verbal. Dans la fonction du prédicat nominal apparaissent des substantifs et des adjectifs sans auxiliaire. Par conséquent, le prédicat nominal est dépourvu d'affixes indiquant le mode, l'aspect et la voix, qui n’apparaissent que dans lé système du verbe. En plus, les formes verbales en fonction du prédicat contiennent une des trois séries de préfixes pronominaux: la série transitive, intransitive ou statique.

$1^{\circ} \mathrm{La}$ racine du prédicat transitif est précédée d'un préfixe objectif et suivie d'un suffixe subjectif indiquant l'agent: $(o-P-a)$. Les préfixes objectifs distinguent trois personnes deux genres, masculin et féminin: $1^{\text {ère }} \mathrm{p}$. ?i$2^{\mathrm{e}}$ p. masc. $w i-$, fém. $h i^{-}, 3^{\text {e }}$ p. masc. ?u-, fém. $t i^{-}$(au singulier). Dans la $3^{\mathrm{e}} \mathrm{p}$. du masculin, il y a trois nombres: singulier, duel et pluriel, mais dans les autres personnes, on ne distingue que deux nombres, c.-̀̀-d. le singulier et le pluriel. Les suffixes subjectifs apparaissent sous la forme de désinences personnelles, p. ex. dans l'aspect momentané: sing. lère p. - ni, $2^{\text {e }}$ p. masc. - $i$-, fém. $-? q, 3^{\text {e }}$ p. masc. $-w i$, fém. $t i$; il y a des paradigmes parallèles pour le duel et pour le pluriel. Dans la même fonction on emploie aussi des formes du verbe auxiliaire ?u'hki he is, was; he exists, existed', à savoir sing. $1^{\text {ère }} p$. 
?a-hkini, $2^{\mathrm{e}}$ p. masc. wi-hki, fém. hi-hki, $3^{\mathrm{e}}$ p. masc. ?u'-hki, fém. ?a-ki, p. ex. ?i-h-pề k-?uhki he has hit me' (गi-'me' préfixe objectif, $h$ - consonne euphonique, pêk- to hit', -?uhki he' auxiliaire dans la fonction du suffixe subjectif de la $3^{\mathrm{e}} \mathrm{p}$. du masc.; H. 356). En tunica, l'agent nominal et l'objet, qui fonctionnent comme appositions aux affixes pronominaux du prédicat transitif, apparaissent dans la forme appositionnelle caractérisée par les désinences du masculin sing. -ku, duel -à'nima, plur. -sềma, ou du féminin sing. -hči, plur. -sínima, p. ex. to'niku the man' (forme appositionnelle masc. sing. en -ku dérivée du mot $t$-jolni a person', $t$ - article); ta'nisarahč 'the girl' (forme appositionnelle fém. sing. en -hči dérivée du mot ta'-ni'sara a young person', ta-article). Le nombre et le genre de la désinence appositionnelle de l'agent et du suffixe subjectif du prédicat dépendent du nombre et du genre de l'agent et analogiquement le nombre et le genre de la désinence de l'objet et du préfixe objectif sont déterminés par le nombre et le genre de l'objet. Par conséquent, dans les propositions où l'agent et l'objet ne sont pas du même nombre et genre, on peut les reconnaître suivant leurs liens grammaticaux avec les affixes pronominaux du prédicat, p. ex. ?ồkaši tohk ?illin ?unra'n?akến 'she raised two boys' lôk kašiltoh-ku 'boy' objet, -ku désinence appositionnelle masc. sing.; îl li two'; ?un-ra'n-?akê'ni prédicat en quotatif caractérisé par l'intonation basse de la dernière voyelle - $i$; ?un- préfixe objectif à la $3^{\text {e }}$ p. duel. masc., lié a l'objet 'two boj s'; - ?akê ni $\leqslant$-?aki-a ni, ?aki she' auxiliaire dans la $3^{\mathrm{e}} \mathrm{p}$. sing. fém. indiquant l'agent, -ani particule enclitique du quotatif; B. Vol. IV., p. 95). L'accord en genre (masculin) et en nombre (duel) entre le groupe syntaxique ?ốkašiltohk ?illín 'two boys' et le préfixe objectif du prédicat ?un- détermine ce groupe comme objet. Au contraire, dans les propositions où l'agent et l'objet sont de même nombre et genre, il n'y a que l'ordre des mots qui permet de les distinguer: $(A)-(O)-(o-P-a)$, p. ex.

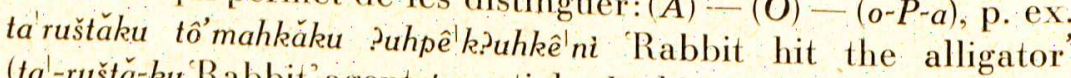
( ta-ruštă-ku Rabbit' agent, ta-article,- $k u$ désinence appositionnelle masc. sing., l'intonation escendante de la voyelle $a$ en ruštăest déterminée par la position devant le prédicat; tố-mahk $\hat{a}^{\prime}-k u$ 
'alligator' objet possédant la structure morphologique parallèle à la structure de l'agent; ?u-h-pê' $k$-?uhkêlni prédicat en quotatif caractérisé par l'intonation basse de la dernière voyelle - $i$; ?upréfixe objectif à la $3^{\mathrm{e}}$ p. sing. masc. lié à l'objet; -juhkềni $\leqslant$ -3uhki-a ni, ?uhki auxiliaire dans la $3^{\mathrm{e}} \mathrm{p}$. sing. masc. indiquant l'agent, -ani particule enclitique du quotatif; $H$. 362).

$2^{\circ}$ Le prédicat intransitif ne contient que le suffixe subjectif indiquant l'agent: $(P-a)$, p. ex. paltawi he fell' ( $p a^{\prime} t a^{-}$to fall', wi la désinence subjective de la $3^{\mathrm{e}}$ p. sing. masc.; $\left.H .355\right)$. Dans la proposition intransitive: $(A)-(P-a)$ la désinence subjective du prédicat s'accorde en genre et en nombre avec l'agent analogiquement que dans la proposition transitive, of. proposition intransitive: ta'nisarăhč ha'rakati 'the girl sings' ( $t a$ 'nisară-hč the girl' agent élargi par la désinence appositionnelle fém. sing. - $h c ̌ \leq-h c ̌ i ; ~ h a ' r a-k a t i$ prédicat contenant le suffixe subjectif $-k a-t i$ de la $3^{\mathrm{e}} \mathrm{p}$. fém. sing. qui s'accorde en genre et nombre avec l'agent; $H .363$ ).

$3^{\circ}$ Le prédicat statique désignant une particularité ne contient que le préfixe objectif indiquant l'objet intransitif qui correspond au sujet intransitif des langues subjectives: $(o-P)$,

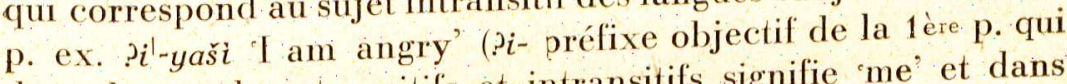
dans les verbes transitifs et intransitifs signifie 'me' et dans les verbes statiques T'; $-y a^{\prime}{ }^{\prime} s i$ to angry'; H. 356). Les verbes auxiliaires possèdent la même structure morphologique que les verbes statiques, p. ex. ?u-hki 'he is' (?u- préfixe objectif de la $3^{\mathrm{e}} \mathrm{p}$. sing. masc. signifiant ici he', H. 349). Dans la proposition statique du type $(O)-(o-P)$, le préfixe objectif du prédicat s'accorde en genre et en nombre avec l'objet nominal (sujet logique), p. ex. ta'ruštǎku ?u'šsêpa'ni lit. 'Rabbit - he was glad' (ta'rušt ǎ-ku Rabbit' objet intransitif élargi par la désinence appositionnelle sing. masc. $-k u$; ?u-šs?êp-a ni prédicat, ?u- préfixe objectif de la $5^{\mathrm{e}} \mathrm{p}$. sing. masc. lié à l'objet, -šsêp - to be glad", $-a^{\prime} n i$ particule enclitique du quotatif; H. 362).

Dans le groupe nominal, le nom du possesseur, élargi par la désinence appositionnelle, fait apposition au préfixe objectif du nom de la chose possedée: $(P r)-(o-P e)$, p. ex. to'nik ?u'rihč 'the man's house', lit. 'the man his house' (to'nik 
de to'ni-ku 'man' possesseur, -ku désinence appositionnelle; ?u-rihč his house', ?u- préfixe objectif de la $3^{\mathrm{e}} \mathrm{p}$. sing. masc. dans la fonction du pronom possessif, s'accordant avec le nom du possesseur -rihč house'; H. 363).

En tunica les mots désignant l'agent, l'objet et le possesseur apparaissent dans la même forme caractérisée par un suffixe qui détermine leur fonction comme appositions aux affixes pronominaux du prédicat ou du nom de la chose possédée. Particulièrement la construction de l'objet transitif, de l'objet statique (le tunica est une langue objective!) et du possesseur est identique, car tous ces membres de la phrase constituent des appositions aux préfixes objectifs et ne sont différenciés que par la signification des mots contenant ces préfixes. L'apposition au préfixe objectif est interprétée comme objet transitif devant les prédicats transitifs, comme objet statique devant les prédicats statiques et comme possesseur devant un nom. A ces multiples fonctions de préfixes objectifs s'oppose une unique fonction du suffixe subjectif n'indiquant que l'agent. Par conséquent, le suffixe subjectif fonctionne en tunica en tant que terme positif de l'opposition, et le préfixe objectif comme terme négatif.

La même structure de la phrase qu'en tunica, c.à-d. purement concentrique, apparaît en chinook ${ }^{2 i}$ ) parlé sur les deux rives de la rivière Columbia (Orégon), en aval de The Dalles jusqua la mer. Le prédicat - unique membre constitutif de la phrase - est caractérisé en chinook par des affixes du mode, du temps et de l'aspect. En plus, le prédicat transitif contient des préfixes pronominaux indiquant l'agent $(a)$, l'objet direct (o) et l'objet indirect $\left(o^{\prime}\right)$. La fonction des préfixes pronominaux est partiellement determinée par leur forme, car dans la $3^{\mathrm{e}} \mathrm{p}$. il y a deux séries de préfixes: la série subjective, indiquant l'agent, et la série objective pour l'objet direct et indirect. En plus, la fonction des préfixes est signalée par leur position. Le premier préfixe pronominal indique l'agent, le deuxième l'objet direct, le troisième l'objet indirect:

27) Chinook, by Franz Boas, B., p. 559-677. 
(a-o-o-P.tr.) p. ex. $a-m-L-a-x-c g-\bar{a} m-x$ thou wert in the habit of taking it from her ( $a$ - préfixe d'aoriste; $-m$ - thou' agent; $-L$ 'it' objet direct; $-a^{-}$her préfixe objectif de la $3^{\mathrm{e}} \mathrm{p}$. fém: dans la fonction de l'objet indirect; $-x$ - préfixe indiquant la relation de la possession entre l'agent et les objets qui signale ici que lobjet direct 'it' appartient à l'objet indirect 'her'; -cg- to take racine; -am suffixe indiquant la direction de laction; $-x$ suffixe déterminant que l'action est répétée par habitude; $B$. 576). Les préfixes pronominaux de la lère et 2 e $p$. distinguent trois nombres (1 ère p. sing. $n$-, excl. duel $n t$-, excl. plur. $n t c$, incl. duel $t x$, incl. plur. $l x-; 2^{\mathrm{e}} \mathrm{p}$. sing. $m$-, duel $m t^{-}$, plur. $m c-$ ). A la $3^{\mathrm{e}}$ p. il y a également trois nombres et en singulier on distingue trois genres, masculin, féminin et neutre (sing. masc, subjectif $t c^{-}$, objectif $i$-; fém. subj. g-, obj. $a^{-;}$, neutre $L_{-}$; duel subj. $c^{-}$, obj. $c^{-}$ou $c^{-}$; plur. $t^{-}$. La fonction syntaxique du prédicat est augmentée encore par son préfixe - $x$-qui indique la relation de la possession entre lagent et les objets. Si ce préfixe apparaît après le premier préfixe objectif, il indique que l'objet direct appartient au sujet. Au contraire, si le préfixe $-x$ - est placé après le second préfixe objectif, l'objet indirect appartient à l'objet direct (cf. le dernier exemple).

Lagent nominal, l'objet direct et indirect ne sont que des appositions aux préfixes pronominaux du prédicat qui saccordent avec eux en nombre et en genre. Par conséquent, dans les propositions transitives du type: $(\alpha-o-o-P . t r)-.(A)$ - $(O)-\left(O^{i}\right)$, on peut reconnaitre la fonction de chaque membre nominal de la phrase d'après la fonction du préfixe pronominal avec lequel il est lié par le fait de l'accord grammatical, p. ex. aqiō cgam iLä'xanate 'bis soul is taken', lit. 'someone takes it - his soul' $\left(a-q-i-\bar{o}^{i}-c g-a m\right.$ 'someone takes it' prédicat, $a$ - préfixe d'aoriste, $-q$ - agent indéfini, $-i$ - préfixe objectif de la $5^{\mathrm{e}} \mathrm{p}$. sing. masc. indiquant l'objet, $-\bar{o}$-directif, $-c g$ - to take' racine,-am suffixe indiquant la direction de l'action; $i-L \bar{a}^{-}$-xanaté his soul' objet s'accordant en genre et en nombre avec le préfixe objectif du prédicat -i-, $i-k a n a ̄ l t e ́$ 'soul', $-L \bar{a}$ - préfixe possessif

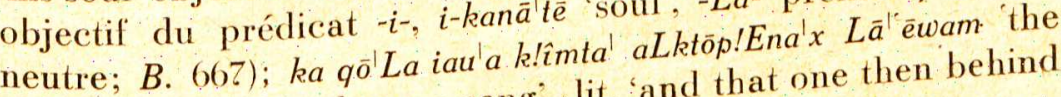
first one utters his shaman song', lit. 'and that one then behind 
he utters it his song' ( $k a$ 'and'; qō $L a$ that one' démonstratif neutre en fonction du sujet indépendant; iaula then'; klı̂mta ‘behind' adverbe; $a-L-k-t-o-p ! E n a '-x$ he utters it' prédicat, $a-$ aoriste; $-L$ préfixe de la $3^{\mathrm{e}}$ p. sing. neutre dans la fonction du préfixe subjectif, ce qui est indiqué par le morphème suivant $-k_{-}$, le préfixe $-L-$ s'accorde en genre et en nombre avec le sujet $q \bar{o} L a ;-t$ - préfixe de la $3^{\mathrm{e}} \mathrm{p}$. plur, dans la fonction du préfixe objectif s'accordant avec l'objet placé après le prédicat; -ō directif; -p!Ena to utter' racine; - $x$ suffixe de l'action habituelle; $L \bar{a}^{\prime}-\bar{e} w a m$ his song' objet lié au préfixe objectif du prédicat $-t$-, $L \bar{a}-$ possessif neutre, $L-q \bar{e}^{-1}$ wam shaman's song'; $B$. 667).

Le prédicat intransitif contient le préfixe objectif indiquant l'objet intransitif (sujet intransitif des langues subjectives) et, en certains cas, le prélixe de l'objet indirect: (o-o-P.intr.), p. ex, $a-y-o^{-}-x n^{2}$ the drifted ( $a-$ aoriste; $-y$ - de $-i$ - devant voyelle he', préfixe objectif de la $3^{\text {e }}$ p. sing. masc dans la fonction de lobject indirect, $\bar{o}_{-}^{-}$directif; $-x E n \bar{e}$ to drift' racine; $B$. 582). Dans la proposition intransitive: $\left(o^{-}\right.$
$o$-P.intr. o préfixe $-(O)$, lobjet intransitif fait apposition au premier

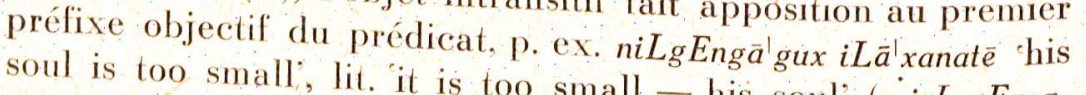
ago-x it is too small prédicat, $n$ - aoriste soul' $(n-i-L-g E n-g-$ de la $3^{\mathrm{e}} \mathrm{p}$. sing. masc. saccordant avec l'objet ${ }^{-}$- préfixe objectif objectif de la 3 e p. sing. neutre dans la fonct soul', $-L$ - préfixe indirect indiquant le propriétaire du premier on account of préfixe adyerbial; - $g$-racine; - ago- around; - $x$ action habituelle; $i-L \bar{a}^{\prime}$ xanate his soul' objet intransitif, lié au premier préfixe objectif; $B$. 669). Le chinook est une langue objective, car l'objet transitif et l'objet intransitif sont caractérisés ici de la même manière.

Dans le groupe nominal (pos.Pe) - $(P r)$, le nom du possesseur $(P r)$ fait apposition au préfixe possessif (pos.) du nom de la chose possédée $(P e)$, p. ex. iLā'xanatè $L k \bar{a}^{\prime}$ nax the soul of a chief', lit. 'his' soul a chief" (i-Lalxanate his soul', $i-k a n a ̄ t e \bar{e}$ soul', -Lä- his' préfixe possessif neutre s'accordant avec le genre neutre du possesseur; $L-k \bar{a}$ ' nax 'a chief' le possesseur indéfini et par conséquent dans le genre neutre; B. 666). 
\$20. Le schème de la proposition transitive composée du sujet, de l'objet et du prédicat est la plus importante construction syntaxique dans toutes les langues du monde. Dans la plupart des langues de l'Amérique du Nord, cette proposition possède la structure concentrique. Ce n'est que dans la famille penutia et dans les langues voisines quapparaît la proposition de la structure excentrique, où chaque membre est constitutif. Parmi les langues excentriques, il y a un groupe de dialectes (yokuts, maidu, hopi et haida) qui caractérisent séparément chaque membre de la proposition par ses affixes ou par sa position dans la phrase. Ces moyens grammaticaux ne possèdent que la fonction syntaxique et n'indiquent aucune direction. Au contraire, dans les langues excentriques et en même temps cycliques (kwakiutl, nass et tsimshian), la fonction dans laquelle apparaît chaque membre de la proposition est déterminée par le dernier suffixe du membre précédent. Dans les langues concentriques la fonction syntaxique est concentrée dans le prédicat à un degré qui varie d'une langue à une autre. Dans les langues concentriques du premier degré (yuma, fox et eskimo), le sujet et l'objet nominaux ont encore une fonction syntaxique. Ils s'opposent réciproquement par leurs désinences qui marquent à quel affixe du prédicat chacun d'eux fait apposition. Dans les langues concentriques du deuxième degré (delaware, chipewyan, tlingit et chitimacha), l’indépendance du sujet et de l'objet nominaux est diminuée, parce qu'ils ne se distinguent que par leur position par rapport au prédicat. Dans les langues du troisième degré (aztèque, dakota), il n'y a pas de moyens syntaxiques pour distinguer le sujet et l'objet qui sont complètement dépourvus de fonction syntaxique, mais en même temps cette fonction n'est pas remplie par le prédicat n'ayant pas de moyens pour distinguer les autres membres de la proposition. Enfin dans les langues du quatrième degré (hupa, taos, tunica et chinook), le sujet et l'objet nominaux sont également dépourvus de fonction syntaxique, mais cette fonction est remplie par le prédicat qui, grâce à la concordance en genre et en nombre de son affixe subjectif au sujet 
nominal et de son affixe objectif à l'objet, détermine la fonction d'éléments nominaux de la proposition. D'un autre point de vue, on peut classer les langues concentriques en synthétiques et analitiques. Dans les langues synthétiques (yuma, chitimacha et taos), on ne voit qu'une série indifférenciée d'affixes pronominaux, qui dans le prédicat transitif expriment en même temps le sujet et l'objet. Au contraire, dans toutes les autres langues concentriques, qui ont la structure analytique, il y a au moins deux séries indépendantes d'affixes pronominaux, la série subjective et la série objective.

Le schème de la proposition intransitive peut être interprété comme forme réduite du schème de la proposition transitive. Dans les langues subjectives, les trois membres de la proposition transitive, c,-ì-d. le sujet (l'agent), l'objet et le prédicat, sont réduits à deux: le prédicat et le sujet. Au contraire, dans les langues objectives, ils sont reduits au prédicat et l'objet qui correspondent logiquement au sujet des langues subjectives. Voici ces schèmes syntaxiques:

La proposition: Les langues subjectives:

transitive:

intransitive:

sujet - prédicat - objet

sujet - prédicat
Les langues objectives:

agent - prédicat - objet prédicat - objet

Dans les langues subjectives, dans l'opposition sujet: objet, l'objet, qui n'apparaît que dans les propositions transitives, fonctionne comme terme positif moins répandu et le sujet comme terme négatif plus répandu. Au contraire, dans les langues objectives, dans l'opposition agent : objet, l'agent a la fonction d'un terme positif, car il n'apparaît que dans la proposition transitive, et l'objet a la fonction d'un terme négatif plus répandu. Le terme négatif de l'opposition est souvent caractérisé par la désinence zéro. Par conséquent, les formes sans désinences fonctionnent dans les langues subjectives comme sujet (yokuts, hopi) et dans les langues objectives comme objet (eskimo).

L'opposition des langues subjectives et objectives, basée sur la relation des propositions intransitives avec les transitives est indépendante de l'opposition des langues excentriques et 
concentriques, liée à la structure des propositions transitives. En réalité, dans tous les types des langues excentriques et concentriques, on trouve des dialectes subjectifs et objectifs:

\begin{tabular}{|c|c|c|c|c|c|c|}
\hline \multirow{2}{*}{ 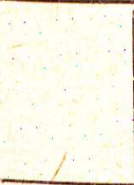 } & \multicolumn{2}{|c|}{$\begin{array}{l}\text { Les langues } \\
\text { excentriques }\end{array}$} & \multicolumn{4}{|c|}{ Les langues concentriques } \\
\hline & $\begin{array}{l}\text { Les Jangues } \\
\text { aux membres } \\
\text { indépendants }\end{array}$ & $\begin{array}{c}\text { Les langues } \\
\text { cycliques }\end{array}$ & 1re degré & 2e degré & ze degré & 4 e degré \\
\hline $\begin{array}{c}\text { Les } \\
\text { langues } \\
\text { subjec- } \\
\text { tives }\end{array}$ & $\begin{array}{c}\text { yokuts } \\
\text { maidu } \\
\text { hopi }\end{array}$ & kwakintl & $\begin{array}{c}\text { yuma(s) } \\
\text { tonkawa(a) } \\
\text { fox }(a)\end{array}$ & $\begin{array}{c}\text { delaware (a) } \\
\text { chipewyan(a) } \\
\text { tlingit(a) }\end{array}$ & $\begin{array}{l}\text { azèque } \\
\text { (a) }\end{array}$ & $\begin{array}{c}\text { apache(a) } \\
\text { hupa(a) }\end{array}$ \\
\hline $\begin{array}{c}\text { Les } \\
\text { langues } \\
\text { objec- } \\
\text { tives. }\end{array}$ & haida & $\begin{array}{c}\text { nass } \\
\text { tsimshian }\end{array}$ & $\operatorname{eskimc}(\mathrm{a})$ & $\begin{array}{l}\text { chitima-: } \\
\text { cha(s) }\end{array}$ & dakota(a) & $\begin{array}{c}\operatorname{taos}(\mathrm{s}) \\
\text { tunica(a) } \\
\text { chinook(a) }\end{array}$ \\
\hline
\end{tabular}

Dans les langues de I'Amérique du Nord, l'objet indirect apparaît dans deux formes, casuelle ou objective. Dans quelques dialectes, il est caractérisé au moyen des désinences casuelles qui remplissent en même temps différentes fonctions adverbiales et locales. C"est à ce type de formes qu'appartient l'indirectif en $-n i$ du yokuts et l'allatif du hopi en -mi et de l'eskimo en -ut. En kwakiutl, l'objet indirect est exprimé par l'instrumental. Au contraire, dans la plupart des langues concentriques, l'objet indirect est indiqué au moyen des affixes objectifs qui sont en même temps employés pour exprimer l'objet direct. En aztèque, apache et chinook, le même préfixe objectif indique l'objet direct ou indirect suivant sa position dans le groupe prédicatif. En chitimacha, dakota, fox et tlingit, un morphème ajouté au prédicat détermine la fonction de laffixe objectif en tant que signe de l'objet indirect. En taos il existe un système analogue quoique plus compliqué.

Dans les langues de l'Amérique du Nord, la structure du groupe nominal est parallèle à la structure de la proposition transitive. Dans les langues où les membres de la proposition sont indépendants (yokuts, maidu) ou presque indépendants

28) Abréviations: $s=$ synthétique, $a=$ analytique. 
(tonkawa), le possesseur $(P r)$ est caractérisé au moyen de la désinence du possessif (pos.) et le nom de la chose possédée $(P e)$ est dépourvu de signes morphologiques: (Pr-pos. $)-(P e)$, cf. lat. domus Marci. Dans les langues cycliques (kwakiutl, nass et tsimshian), le premier membre du groupe nominal, indiquant la chose possédée, est élargi par un suffixe syntaxique (pos'.) qui caractérise le mot suivant comme possesseur: $\left(P e-p o s^{\prime}.\right)-(P r)$. Au contraire, dans la plupart des langues concentriques et en plus dans le haida et hopi, le nom du possesseur constitue l'apposition à l'affixe pronominal possessif (pos.) du nom de la chose possédée: $(P r)-($ pos:-Pe), le type le père sa-maison 'la maison du père'. En plusieurs langues excentriques (hopi, nass, tsimshian) et concentriques (chipewyan, hupa, apache, yuma, tunica), la relation du possesseur avec la chose possédée est indiquée au moyen de la même construction que la relation de l'objet avec le prédicat.

Dans les langues nord-américaines, les relations syntaxiques sont indiquées au moyen de trois types de constructions, c.-à-d. des constructions casuelles, des signes grammaticaux employés pour caractériser les membres de la phrase et enfin au moyen des constructions appositionnelles. Dans les constructions casuelles, les relations abstraites entre le sujet, l'objet direct ou indirect et le prédicat, entre le possesseur et la chose possédée, sont indiquées à l'aide des affixes qui, dans le même système linguistique, sont employés pour indiquer certaines directions. Ce type de constructions, très répandu dans les langues casuelles d'Europe et d'Asie, aussi bien subjectives (les langues indo-européennes et chamito-sémitiques) qu'objectives (les langues dites "asianiques”. ou ,yaphétiques“), est extrêmement rare dans les dialectes nord-américains. II n'y a que l'allatif de l'eskimo et du hopi et l'indirectif du yokuts qui peuvent être interprétés comme cas employés pour caractériser l'objet indirect.

Au contraire, des constructions bien connues en Amérique du Nord sont celles dans lesquelles les membres de la phrase sont caractérisés au moyen de signes grammaticaux (affixes du mot, suffixe du mot précédent, ordre des mots) qui n'in- 
diquent que la place du mot dans le schème de la proposition sans aucune valeur locale ou adverbiale. En yokuts, le prédicat, le sujet et l'objet direct sont caractérisés à l'aide des suffixes de ce type et des systèmes analogues fonctionnent en maidu (pour l'objet et le possesseur), en tonkawa et dans les langues cycliques (kwakiutl, nass et tsimshian). En plus, la fonction syntaxique des affixes pronominaux du prédicat dans les langues concentriques est caractérisée de manière analogue. Chacun de ces affixes détermine sa fonction comme signe du sujet ou de l'objet direct ou indirect à l'aide de sa forme ou de sa position et ces moyens sont tout-à-fait dépourvus de la valeur concrète.

Dans les langues nord-américaines, des constructions appositionnelles basées sur la relation du membre déterminant avec le membre déterminé, sont les plus répandues. En haida, le prédicat fonctionne en apposition de l'objet. Au contraire, en hopi, c'est l'objet qui constitue l'apposition du prédicat. En maidu, le sujet détermine la désinence personnelle du prédicat et, dans les langues concentriques, le sujet et l'objet fonctionnent comme appositions des affixes pronominaux du prédicat. Par conséquent, en plusieurs langues concentriques, il n’y a pas de différence entre la forme de l'objet et du possesseur, car l'objet y fait apposition de l'affixe objectif du prédicat et le possesseur de l'affixe objectif-possessif du nom de la chose possédée. Dans ces langues, la structure de la phrase verbale analogiquement à la structure du groupe nominal est basée sur la relation des membres déterminants (sujet, objet, possesseur) et des membres déterminés (prédicat, nom de la chose possédée). Cette grande importance des constructions appositionnelles est caractéristique pour les langues de l'Amérique du Nord. 